\title{
Revision of the elusive ant genus Rhopalomastix (Hymenoptera, Formicidae, Myrmicinae) in Thailand based on morphology and DNA barcodes, with descriptions of three new species
}

\author{
Wendy Y. WANG ${ }^{1, *}$, Gordon W.J. YONG ${ }^{2} \&$ Weeyawat JAITRONG ${ }^{3}$ \\ ${ }^{1}$ Lee Kong Chian Natural History Museum, National University of Singapore, \\ 2 Conservatory Drive, Singapore 117377. \\ ${ }^{2}$ Department of Biological Sciences, Faculty of Science, National University of Singapore, \\ 16 Science Drive 4, Singapore 117558. \\ ${ }^{3}$ Natural History Museum, National Science Museum, Technopolis, Khlong 5, \\ Khlong Luang, Pathum Thani, 12120 Thailand. \\ *Corresponding author: nhmwyw@nus.edu.sg - wywang24@gmail.com \\ 2Email: gordonyongwj@gmail.com \\ ${ }_{3}^{3}$ Email: polyrhachis@yahoo.com \\ ${ }^{1}$ urn:1sid:zoobank.org:author:41730B76-D515-42D0-A3BE-2788ADFDAA8A \\ ${ }^{2}$ urn:1sid:zoobank.org:author:3D3752DF-3522-4DEE-B4A3-7E85CA774263 \\ ${ }^{3}$ urn:1sid:zoobank.org:author:E456F06F-486F-4BD5-AEE6-6DCA639C3E27 \\ $1 \odot$ https://orcid.org/0000-0003-0745-4702 \\ ${ }^{3} \odot$ https://orcid.org/0000-0003-1362-0754
}

\begin{abstract}
The actual prevalence and diversity of the elusive Asian ant genus Rhopalomastix Forel, 1900 in Southeast Asia are largely unknown; only two named species were previously known from Thailand. Following substantial newly-collected museum material made available, we used a combined approach based on morphology and complementary DNA evidence to revise the genus in Thailand. Specimens were sorted to putative species by objective clustering of short fragment (313 bp) COI barcodes, after which specimens of each molecular cluster were morphologically examined. With morphology and supporting genetic evidence, we recognize five species of Rhopalomastix in Thailand, including three species new to science: $R$. impithuksai Wang \& Jaitrong sp. nov., $R$. parva Wang \& Jaitrong sp. nov., and $R$. robusta Wang \& Jaitrong sp. nov. Different castes and sexes are described for most species where available. Descriptions of the two extant described species $-R$. javana Wheeler, 1929 and $R$. johorensis Wheeler, 1929 - are also revised, taking into account observations from the additional material. A key to Thai species based on the worker caste, with the three new species included, is further provided.
\end{abstract}

Keywords. Systematics, NGS barcoding, arboreal ant, objective clustering.

Wang W.Y., Yong G.W.J. \& Jaitrong W. 2012. Revision of the elusive ant genus Rhopalomastix (Hymenoptera, Formicidae, Myrmicinae) in Thailand based on morphology and DNA barcodes, with descriptions of three new species. European Journal of Taxonomy 739: 117-157. https://doi.org/10.5852/ejt.2021.739.1271 


\section{Introduction}

Rhopalomastix Forel, 1900 is a small-sized Asian myrmicine genus in the tribe Crematogastrini Forel, 1893 (Ward et al. 2015) whose actual prevalence and diversity are poorly understood and largely unknown (Wang et al. 2018b). These ants nest in bark of living trees and are rarely collected via common collecting methods for most other ants, such as leaf litter sifting, honey baiting or pitfall traps. Congeners also tend to appear morphologically similar with no striking differences among species, a problem exacerbated by the ants' minute size. Ants of this genus can be distinguished by unique morphological characters also seen in their Afrotropical cousin Melissotarsus Emery, 1877, such as: 1) median section of clypeus not projecting back between antennal sockest, 2) very short antennae, each with a somewhat flattened funiculus and an expanded, robust club comprising two apical segments, 3) frontal lobes short, narrow and very closely approximated, separated by a small impressed line, 4) mesosoma short and box-like, dorsum without sutures or conspicuous deep impressions, usually finely striated, 5) middle and hind basitarsi short and stout, 6) propodeal lobes absent (Bolton 2003; Wang et al. 2018b). Moreover, both genera are the only ants known to live with scale insects (Hemiptera: Diaspididae) in their nests, with which they seem to share an obligately mutualistic relationship (Peeters et al. 2017; Yong et al. 2019).

Despite their apparent similarities, Rhopalomastix and Melissotarsus also have myriad differences. First, the two genera have completely discrete geographical distributions - Rhopalomastix is found in the Oriental and Indo-Australian tropics, whereas Melissotarsus is limited to the Afrotropics. Rhopalomastix can also be differentiated from Melissotarsus based on multiple morphological traits, for example: Rhopalomastix has ten antennal segments, Melissotarsus has six; Rhopalomastix has procoxa as large as or larger than remaining coxae, in Melissotarsus the procoxa is much smaller than other coxae; the basitarsus is not swollen in Rhopalomastix, but in Melissotarsus the meso- and metabasitarsi are swollen with apical circlets of short traction spines or teeth (Bolton 2003; Wang et al. 2018b). Overall, Rhopalomastix seems to be more 'generalized' in morphology with less conspicuously developed or specialized characters (Bolton 2003). Furthermore, while both genera have been found to exhibit silkspinning behaviour (Peeters et al. 2017; Yong et al. 2019), and possess silk-producing glands in their heads (Billen \& Peeters 2020), Rhopalomastix appears to have silk glands with relatively fewer secretory cells and lower secretory activity (Billen \& Peeters 2020).

Currently, eleven species are recognized in the genus. Among these, only two species - R. javana Wheeler, 1929 and R. johorensis Wheeler, 1929 - have been recorded specifically from Thailand (Wang et al. 2018b). Using a combined taxonomic approach starting with rough sorting into putative species units based on short fragment mitochondrial DNA barcodes, followed by detailed morphological examination, we investigated new museum material comprising nest series of unidentified Rhopalomastix species collected across various parts of Thailand. We compared this new material with type and non-type specimens of known species. We identified and herein describe an additional three new species from Thailand, supported by morphology and DNA evidence; we also revise and correct (where necessary) descriptions of the current known species taking into account observations from newly identified material. A key to Rhopalomastix species in Thailand based on the worker caste is provided.

\section{Material and methods}

This study is mainly based on material deposited in the Zoological Reference Collection (ZRC) of the Lee Kong Chian Natural History Museum (Singapore), and the Natural History Museum of the Thai National Science Museum (Thailand). Morphological observations were made using an Olympus SZX16 stereo microscope, while measurements were made using micrometres on the same microscope. All measurements are given in millimetres and recorded to the second decimal place. 


\section{Abbreviations used for measurements and indices}

\section{Measurements}

$\mathrm{EL} \quad=$ Maximum eye length measured along its maximum horizontal diameter

$\mathrm{EW}=$ Maximum eye width perpendicular to EL

$\mathrm{HL}=$ Maximum head length in full-face view, measured in a straight line, from the anterior clypeal margin to the midpoint of a straight line drawn across the posterior margin of the head

HW $=$ Maximum head width behind the eyes, measured in full-face view

ML = Mesosomal (Weber's) length, measured as the diagonal length of the mesosoma in profile, from the anteriormost point at which the pronotum meets the cervical shield to the posterior basal angle of the metapleuron

$\mathrm{MsW}=$ Maximum width of the mesoscutum (queens and males only) in dorsal view

PronW $=$ Maximum width of pronotum in dorsal view

$\mathrm{PtH}=$ Petiole height, measured in profile view and at a right angle to PL, taken from dorsal surface of petiolar node to ventral surface of postpetiolar helcium, where it inserts into petiole

$\mathrm{PtL}=$ Petiole length, measured in profile view from the anterior margin of the peduncle to posteriormost point of the petiolar tergite

$\mathrm{SL}=$ Maximum length of the antennal scape measured in a straight line, excluding the basal constriction and condylar bulb

$\mathrm{TL}=$ Total body length, roughly measured in profile view from the anterior margin of the head to the apex of the gaster for outstretched specimens

\section{Indices}

$\mathrm{CI}=$ Cephalic index, $\mathrm{HW} / \mathrm{HL} \times 100$

$\mathrm{PtHI}=$ Petiolar Height Index, PtH/PtL $\times 100$

$\mathrm{REL}=$ Relative eye length, $\mathrm{EL} / \mathrm{HW} \times 100$

SI $=$ Scape index, $\mathrm{SL} / \mathrm{HW} \times 100$

Specimens were imaged with a Dun Inc. ${ }^{\mathrm{TM}}$ Passport II macrophotography imaging system, using a Mitutoyo 7D MKII objective lens with $10 \times$ or $20 \times($ for closeup profiles of head $)$ zoom. Focus-stacked images were produced using Zerene Stacker (Zerene Systems LLC, http://zerenesystems.com/cms/stacker). The final images were annotated, and scale bars added using Adobe ${ }^{\circledR}$ Photoshop CS6.

\section{Abbreviations of specimen depositories}

$\mathrm{MCZ}=$ Museum of Comparative Zoology, Harvard University, Cambridge, Massachusetts, USA

THNHM $=$ Natural History Museum of the National Science Museum, Pathum Thani, Thailand

USNM = Smithsonian Museum of Natural History, Washington D.C., USA

ZRC $=$ Zoological Reference Collection, Lee Kong Chian Natural History Museum, Singapore

\section{DNA extraction}

DNA extraction for each representative specimen of the new museum material was performed using QuickExtract ${ }^{\mathrm{TM}}$ DNA extraction solution (Kranzfelder et al. 2016), according to the manufacturer's instructions. The extracts were used as templates for subsequent COI (313 bp) barcoding.

\section{DNA Barcoding and putative species sorting}

PCR amplification and next generation sequencing of the barcodes were performed following procedures described in Wang et al. (2018b). Successful barcodes were checked for contamination against the GenBank (NCBI) nucleotide database (Benson et al. 2013), using the online NCBI Basic Local Alignment Search Tool (BLAST) ver. 2.6.0+ (Altschul et al. 1990) under default parameters in Megablast (word size: 28). Verified barcodes were then aligned with barcodes identified from known 
Rhopalomastix species in Thailand, mostly generated in a previous study by the same authors (i.e., Wang et al. 2018a) (2-3 representative barcodes selected from each colony of named species examined), using MAFFT ver. 7 (Katoh \& Standley 2013), and alignments were checked on MEGA 6 (Tamura et al. 2013). The numbers of molecular operational taxonomic units (mOTUs) - or putative species clusters - over a range of multiple percentage thresholds were determined using a custom-built Python scriptbased software obj_clust ver. 0.1.2 (see Wang et al. 2018b). The barcodes were grouped by 'objective clustering' - where sequences are grouped according to uncorrected pairwise or $p$-distances using the 'best close match' criteria (Meier et al. 2006, 2008). Under the 'best close match' criteria, members of a set of putative conspecific sequences have at least one match to another sequence in that set, which falls within a given percentage distance threshold. Cluster splitting and/or merging events amongst barcode sequences were visualized using the same customized software.

\title{
Results
}

\section{DNA barcoding and putative species sorting}

A total of 86 COI barcodes from specimens collected across Thailand (North, South, East, West) were clustered into putative molecular species, including eight sequences successfully obtained from the three morphologically identified new species, broken down as follows: $R$. impithuksai sp. nov. -4 ; $R$. parva sp. nov. $-2 ; R$. robusta sp. nov. -3 . For the remaining two named species, 33 barcodes across 11 colonies for $R$. javana, and 44 barcodes across 15 colonies generated for $R$. johorensis, were included in the clustering. All five species were clearly differentiated from each other in terms of COI (313 bp) distance, interspecies divergences in the range of $5.4-15.4 \%$ uncorrected $p$-distance (Fig. 1). Sequences from $R$. parva Wang \& Jaitrong sp. nov. were divergent from the other four species by $15.4 \%$ uncorrected $p$-distance. Rhopalomastix javana diverged from $R$. impithuksai Wang \& Jaitrong sp. nov., $R$. johorensis and $R$. robusta Wang \& Jaitrong sp. nov. at $14.4 \%$ threshold distance. Variation amongst sequences of $R$. javana from different colonies was quite broad $-0.0-4.5 \%$, suggesting a possible underlying species complex or multiple sibling species. Next, $R$. johorensis diverged from $R$. impithuksai Wang \& Jaitrong sp. nov. and $R$. robusta Wang \& Jaitrong sp. nov. at 13.1\% threshold. One group of East Thai specimens morphologically identified as $R$. johorensis, differed from all other $R$. johorensis by $4.5 \%$, once again suggesting possible sibling species. Finally, between two of the three new species, $R$. robusta Wang \& Jaitrong sp. nov. differed from $R$. impithuksai Wang \& Jaitrong sp. nov. by $5.4 \%$ uncorrected $p$-distance. Barcode sequences are available from the corresponding author upon request, and also on GenBank (accession no. MW267045 to MW267130).

\section{Taxonomy}

\author{
Class Insecta Linnaeus, 1758 \\ Order Hymenoptera Linnaeus, 1758 \\ Family Formicidae Latreille, 1809 \\ Subfamily Myrmicinae Lepeletier de Saint-Fargeau, 1835 \\ Tribe Crematogastrini Forel, 1893
}

Genus Rhopalomastix Forel, 1900

Rhopalomastix Forel, 1900: 24. Type species: Rhopalomastix rothneyi Forel, 1900, by monotypy.

Rhopalomastix in Myrmicinae, Solenopsidini - Wheeler 1910: 140.

Rhopalomastix in Myrmicinae, Melissotarsini - Emery 1914: 40; 1922: 118. — Forel 1917: 242. — Wheeler 1922: 661. - Bolton 2003: 72.

Rhopalomastix in Myrmicinae, Crematogastrini - Ward et al. 2015: 71, 77. 


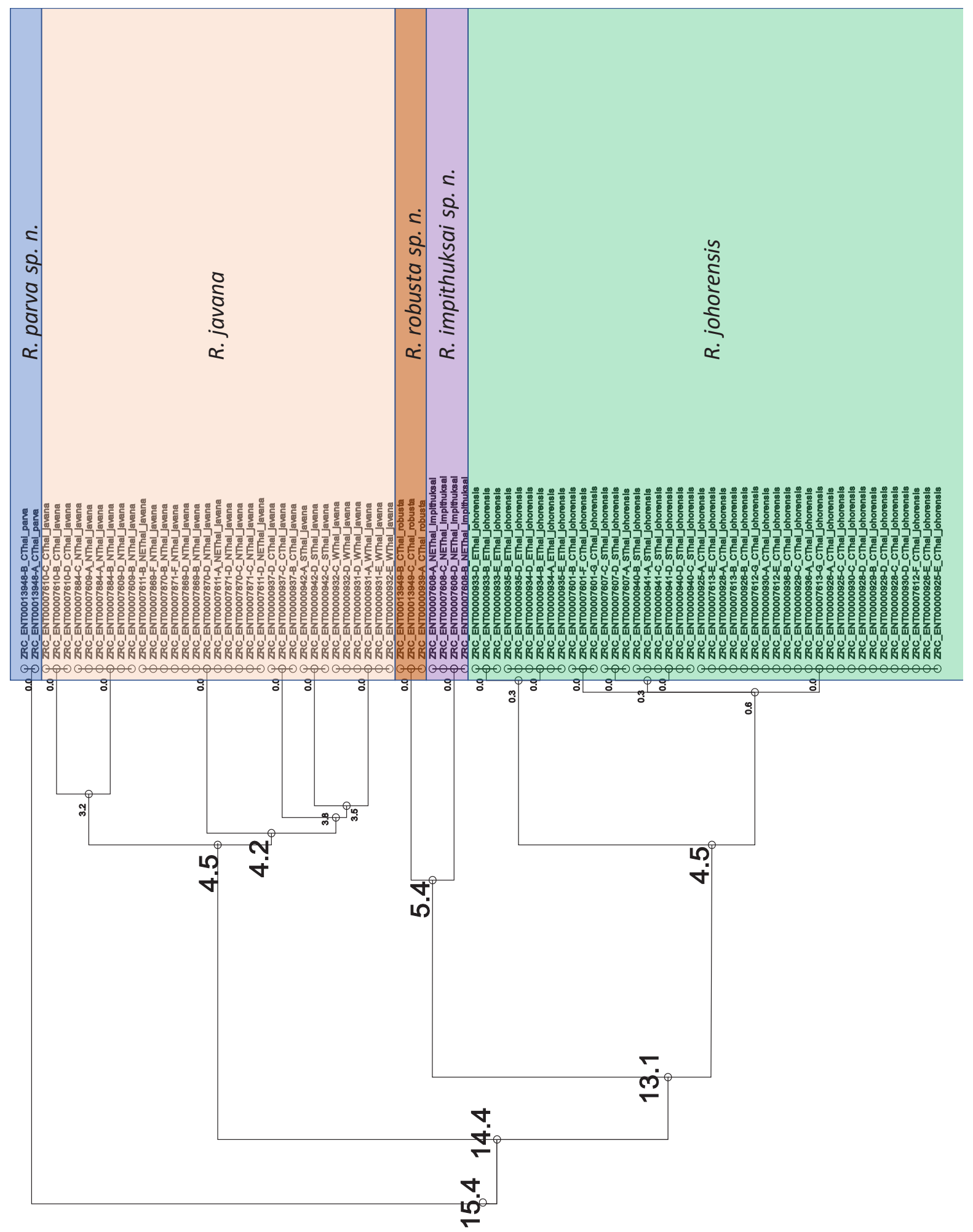

Fig. 1. Cluster dendrogram of COI (313 bp) barcodes of Rhopalomastix Forel, 1900 from Thailand. Objective clustering of barcodes was performed based on uncorrected $p$-distances using the 'best close match' criteria; numbers at nodes represent percentage divergences or pairwise distance thresholds at which clusters split or lump together. 
For diagnosis of the workers in the genus Rhopalomastix, refer to Bolton (2003) and Wang et al. (2018b). For diagnoses of the queen and male of the same genus, see Wang et al. (2018b).

\section{Synoptic list of species in Thailand}

Rhopalomastix impithuksai Wang \& Jaitrong sp. nov.

Rhopalomastix javana Wheeler, 1929

Rhopalomastix johorensis Wheeler, 1929

$=$ Rhopalomastix janeti Donisthorpe, 1936

Rhopalomastix parva Wang \& Jaitrong sp. nov.

Rhopalomastix robusta Wang \& Jaitrong sp. nov.

\section{Key to Thai species based on the worker caste}

1. External face of mandible other than narrow strip next to masticatory margin with distinct striations (Fig. 2a-b); extent and intensity of striations variable. Median clypeal area carinulate, supraclypeal area with dark-pigmented longitudinal carinae

- External face of mandible other than narrow strip next to masticatory margin largely smooth and shiny (Fig. 2c-e). Median clypeal area and supraclypeal area with varying forms of sculpture but never with multiple dark-pigmented longitudinal carinae

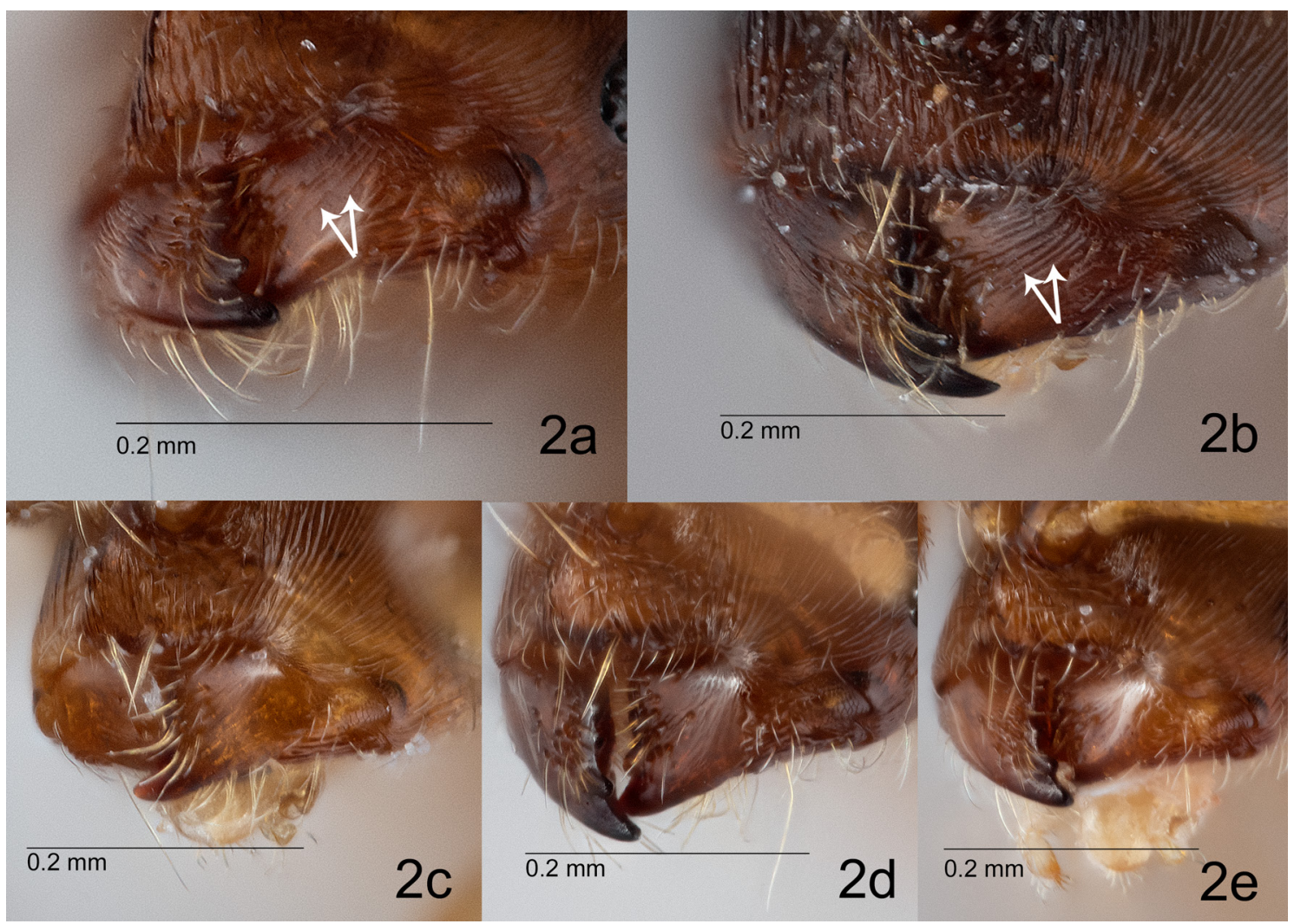

Fig. 2. Closeups of external mandibular surface for workers of 5 Thai species of Rhopalomastix Forel, 1900 (ZRC); white arrows indicate distinct striations on the external mandibular face. a. $R$. impithuksai Wang \& Jaitrong sp. nov., paratype (ZRC_ENT00007608). b. R. robusta Wang \& Jaitrong sp. nov., paratype (ZRC_ENT00013949). c. R. javana Wheeler, 1929, non-type (ZRC_ENT00000932). d. $R$. johorensis Wheeler, 1929, non-type (ZRC_ENT00007874). e. $R$. parva Wang \& Jaitrong sp. nov., paratype (ZRC_ENT00013948). 
2. Striations on dorsum of head uniformly dense from frontal lobes to posterior margin; relatively large species (TL 2.24-3.16), total length of smallest worker $>2 \mathrm{~mm}$. Large worker with head blackish brown, and either as long as wide or wider than long (CI 100-103)

R. robusta Wang \& Jaitrong sp. nov.

- Striations on dorsum of head weaker and more fragmented in posterior half; relatively smaller species (TL 1.84-2.93), total length of smallest worker $<2 \mathrm{~mm}$. Worker (of all sizes) with head dark brown, but never blackish, always longer than wide (CI 94-96)

R. impithuksai Wang \& Jaitrong sp. nov.

3. Head distinctly much longer than wide (CI 84-87); anterior margin of pronotal disc (excluding the collar) strongly convex with roundly angulate median point. Relatively small species (TL 1.852.25)

R. parva Wang \& Jaitrong sp. nov.

- Head not distinctly longer than wide (CI 87-96); anterior margin of pronotal disc (excluding the collar) broadly and often weakly convex with no angular median point 4

4. Propodeal junction in lateral view distinct and roundly obtuse; propodeal dorsum differentiated from posterior declivity by weakly marginate angular edge. Posterolateral corner of propodeal dorsum angulate; posterior propodeal face overall weakly marginate. Anterior clypeal face in profile weakly and broadly convex

R. javana Wheeler, 1929

- Propodeal junction in lateral view indistinct and broadly rounded, propodeal dorsum rounding smoothly into and not differentiated from posterior declivity by marginate angular edge. Posterolateral corner of propodeal dorsum usually indistinct, sometimes bluntly and shallowly rounded; posterior propodeal face overall incompletely marginate with obscure dorsal edge. Anterior clypeal face in profile mostly flat or weakly and broadly convex

R. johorensis Wheeler, 1929

Rhopalomastix impithuksai Wang \& Jaitrong sp. nov. urn:1sid:zoobank.org:act:DC6C24A1-9813-4DED-8B83-150BBCCFA291

Figs 2a, 3 (worker), 4 (male), 16a

\section{Diagnosis}

\section{Worker}

Monomorphic with broad size variation (HL 0.4-0.55; HW 0.44-0.52). Head in full-face view subquadrate, lateral margins broadly convex and nearly parallel (Fig. 3a). In profile, short dorsal clypeal face nearly flat or weakly convex and downward sloping, differentiated from steep and mostly flat anterior clypeal face by indistinct rounded edge (Fig. 3c). Dorsal outline of mesosoma very weakly and broadly convex, almost entirely flat (Fig. 3d). In dorsal view, anterior margin of pronotal disc (thereinafter defined as an area excluding the pronotal collar) broadly convex, humeral corners rounded and indistinctly angulate especially in small worker, lateral margins of propodeum broadly convex with rounded posterolateral corners, posterior propodeal margin indistinct and weakly concave (Fig. 3b). Mandible with upper half of outer face finely striate, lower half mostly smooth and shining (Fig. 2a). Median clypeal face carinulate, interspaces punctate and weakly shiny; supraclypeal area with darkpigmented carinae (Fig. 3a). Narrow vertexal area adjacent to and along posterior margin of head without standing hairs, hairs on gena sparser posteriorly, posterolateral face with sparse or no hairs. Anterior rising face of petiole with sparse and few decumbent and standing hairs, mostly close to apex. Vertexal area of head dorsum anterior to median concavity of posterior margin darker blackish-brown compared to rest of head (Fig. 3a). 


\section{Male}

Moderately large size. Head in full-face view subcircular, distinctly wider than long with broadly convex posterior margin (Fig. 4a); in lateral view, head dorsum almost flat, ventral margin strongly convex without distinct posteroventral angle (Fig. 4b); dorsal clypeal face rounding into weakly convex anterior clypeal face at indistinct rounded edge (Fig. 4b). Median ocellus suboval and similar to lateral ocelli in size and shape (Fig. 4a, c). Frontal lobes shallowly raised and indistinctly differentiated from supraclypeal area; posterior apex of supraclypeal area emarginate and continuous with narrow median furrow between frontal lobes (Fig. 4a). In lateral view, anterodorsal face of mesoscutum distinctly higher than anterior face of pronotum (Fig. 4e). In posterior view, posterior propodeal face emarginate, rounding into and undifferentiated from lateral propodeal face.

\section{Etymology}

The species is named after Dr Viroch Impithuksa, whose assistance was vital for the discovery of the former.

\section{Material examined}

Holotype

THAILAND • worker; Northeast Thailand, Sakhon Nakhon Province, Phu Phan Ratchaniwet Palace; 17 Jun. 2018; W. Jaitrong leg.; nest in bark of mango tree; colony code: WJT170618-5; THNHM-I-21751.

\section{Paratypes}

THAILAND • 7 workers; same collection data as for holotype; THNHM-I-21752 to THNHM-I-21758 • 6 workers, 1 \% ; same collection data as for holotype; GenBank: MW267045 to MW267048 (4 workers barcoded); ZRC_ENT00007608.

\section{Description}

\section{Worker}

Measurements. Paratype workers ( $\mathrm{n}=5$ ): EL 0.07-0.1; EW 0.05-0.06; HL 0.47-0.55; HW 0.44-0.52; ML 0.54-0.70; PronW 0.28-0.36; PtH 0.16-0.20; PtL 0.17-0.22; SL 0.18-0.20; TL 1.84-2.30; CI 9496; PtHI 84-100; REL 16-21; SI 38-41.

Monomorphic workers with broad intranidal size variation. Head in full-face view subquadrate, slightly longer than wide, in large worker head wider posteriorly, in small worker width of head mostly uniform throughout entire length; posterior margin broadly and weakly convex with a shallow median concavity, posterolateral corners roundly convex, lateral margins of head weakly convex and nearly parallel; median furrow short, distinctly much wider and more shallow in posterior half; anterior clypeal margin with broadly convex median section (Fig. 3a). Eye with 12-17 ommatidia. Supraclypeal area with darkpigmented longitudinal carinae, posterior apex carinate and acute, distinctly inserted between frontal lobes and raised above deep anterior end of median furrow. In lateral view, clypeus projecting strongly from dorsal margin of head, short dorsal clypeal face nearly flat or weakly convex and downward sloping, differentiated from steep and mostly flat or weakly convex anterior clypeal face by indistinct rounded edge (Fig. 3c). Mesosoma in lateral view box-shaped or subcylindrical (generally in smaller workers), dorsal outline very weakly and broadly convex, almost entirely flat, propodeal junction roundly obtuse, propodeal declivity steep and shallowly concave; propodeal dorsum differentiated from posterior declivitous face by weak marginate edge (Fig. 3d). In dorsal view, mesosoma subrectangular, anterior margin of pronotal disc broadly convex, humeral corners rounded and indistinctly angulate especially in smaller workers, lateral margins of propodeum broadly convex, transitioning to posterior propodeal margin at bluntly rounded posterolateral corners; posterolateral corners weakly projected posteriorly and shallowly differentiated from inner poster propodeal face; posterior propodeal margin indistinct and weakly concave (Fig. 3b). 
Posterior face of propodeum weakly marginate, rounding into lateral propodeal face at indistinct angulate edge. In lateral view, petiole subtriangular, inclined posteriorly, slightly longer than high or sometimes as long as high, anterior face steep and weakly concave; apex roundly convex, apical dorsum rounding into weakly convex posterior face at indistinct rounded edge, posterior face steeper and shorter than anterior face (Fig. 3d). Petiole higher and longer than postpetiole, dorsal margin of postpetiole weakly convex. In dorsal view, petiole suboval, wider than long; postpetiole globular, less than twice as wide as petiole.

Dorsum of head largely finely substriate and shiny with scattered punctures, lateral face largely superficially substriate-reticulate with scattered punctures and interspaces smooth and shining. Ventral face of posterior half of head mostly superficially reticulate with interspaces smooth and shining. Mandible in full-face view with upper half of outer face finely striate, lower half mostly smooth and shining, area immediately adjacent to masticatory margin punctate (Fig. 2a). Median clypeal face carinulate, interspaces punctate and weakly shiny (Fig. 3a). Lateral face of mesosoma largely substriate-

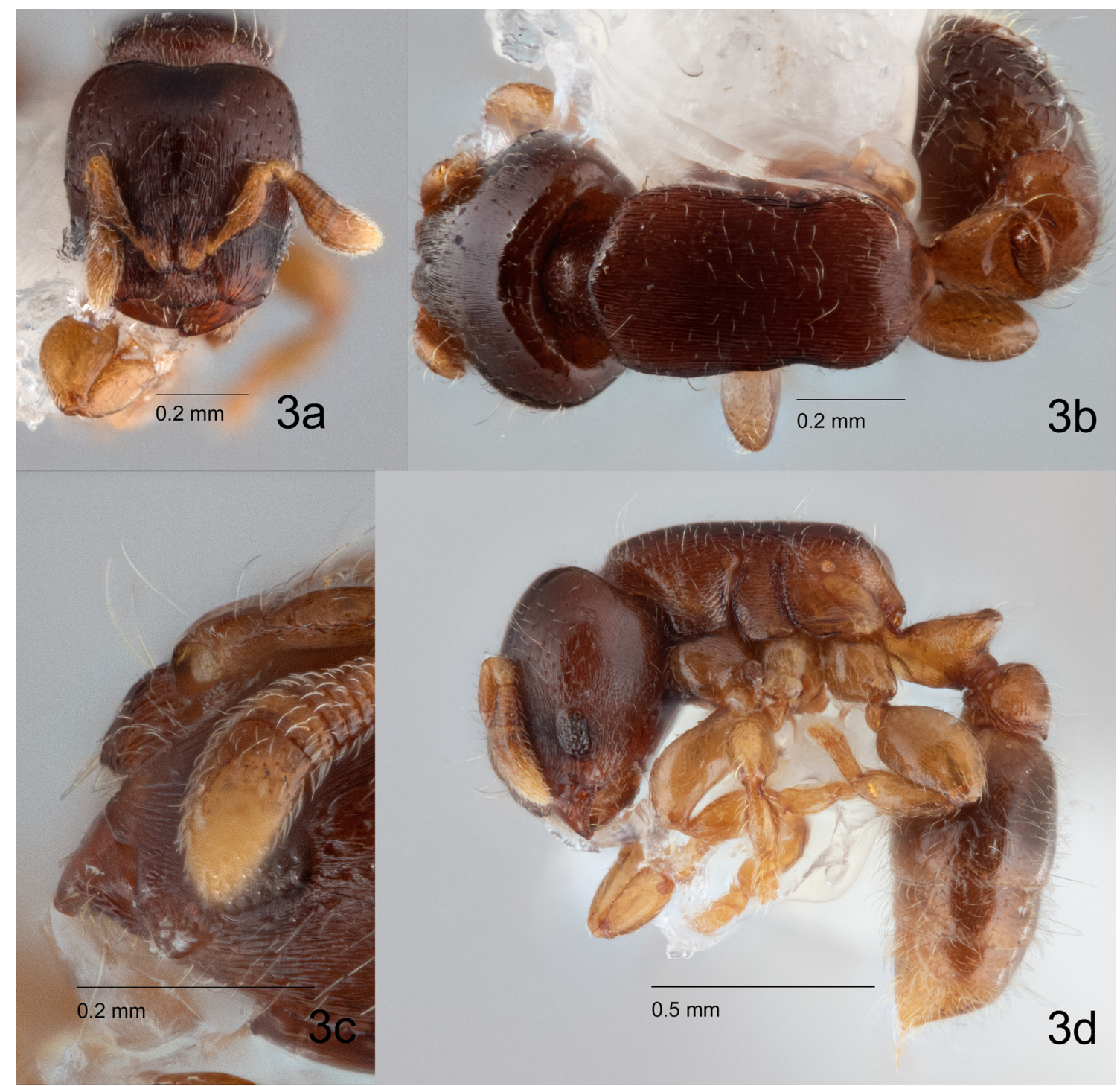

Fig. 3. Rhopalomastix impithuksai Wang \& Jaitrong sp. nov., paratype, worker (ZRC_ENT00007608.1). a. Head in full face view. b. Mesosoma in dorsal view. c. Close-up of clypeus in profile. d. Body in lateral view. Note: anterior face of petiolar node near apex dented/slightly deformed. 
reticulate with interspaces smooth and shining (Fig. 3d); dorsum of mesosoma finely striate and shiny (Fig. 3b); posterior face of propodeum superficially and weakly reticulate with interspaces smooth and shining. Lateral faces of petiole and postpetiole superficially substriate-reticulate with interspaces smooth and shining (Fig. 3d), apicodorsal faces superficially reticulate with interspaces smooth and shining. Gaster superficially reticulate with interspaces smooth and shining, sculpture on first gastral tergite stronger than rest of gaster.

Dorsum of head largely with fairly dense though well-spaced short erect and suberect hairs interspersed with sparse long erect hairs, narrow vertexal area adjacent to and along posterior margin of head without standing hairs, gena with short erect and decumbent hairs that are sparser posteriorly, posterolateral face with sparse or no hairs. Ventral face of head anteriorly with fairly dense short standing and decumbent hairs, sparser standing hairs posteriorly. Mesosoma dorsum with sparse, scattered short standing hairs mostly closer to dorsolateral margin, paired long erect hairs sparsely present along dorsolateral margin, with one pair each flanking the anterior margin of pronotal disc, pronotum, mesonotum and propodeum, sometimes two pairs flanking propodeum. Anterior rising face of petiole mostly hairless with few decumbent and standing hairs close to apex, posterior declivity with dense short standing hairs; apex of petiolar node with few short erect hairs and 1-2 pairs of long erect hairs. Postpetiole with fairly dense short erect and suberect hairs, and one pair of long erect hairs. Gaster pilose with dense erect and suberect hairs, interspersed with sparse long erect hairs.

Head, dorsum of mesosoma and gaster generally dark brown with rest of body more yellowish brown, small worker lighter brown relative to large worker. Vertexal area of head dorsum anterior to median concavity of posterior margin darker blackish-brown compared to rest of head (Fig. 3a), tips of antennae and legs pale yellowish-brown.

\section{Male}

Measurements. One paratype male, antennal funiculi damaged with missing segments ( $\mathrm{n}=1)$ : TL 2.93; HL 0.46; HW 0.48; SL 0.06; PtH 0.20; PtL 0.28; ML 0.90; MsW 0.50; EL 0.24; EW 0.19; CI 104; PtHI 71; REL 50; SI 13.

Moderately large size, similar to $R$. johorensis male. Head in full-face view subcircular, distinctly wider than long, posterior margin broadly convex (Fig. 4a). Eye very large (20-25 ommatidia in the longest axis); posteriormost point of eye slightly exceeding transverse midline of head, outline of eye bulging distinctly from lateral margin of head (Fig. 4a). Median ocellus suboval, wider than long, similar to lateral ocelli in size and shape; area of head dorsum anterior to median ocellus weakly depressed, continuous with weak median furrow separating frontal lobes; frontal lobes shallowly raised and indistinctly differentiated from supraclypeal area (Fig. 4a, c). Posterior apex of supraclypeal area emarginate, continuous with narrow median furrow between frontal lobes (Fig. 4a). In lateral view, head broad-ovate with almost flat dorsum, dorsal face rounding into posterior face at indistinct edge, posterior margin rounding into strongly convex ventral margin forming a continuous curve with no distinct posteroventral angle; clypeus strongly projecting from dorsum of head, dorsal clypeal face gently sloped downwards and nearly flat, differentiated from weakly convex anterior clypeal face by indistinct rounded edge (Fig. 4b).

In lateral view, mesosoma sub-oblong, dorsal margin weakly and broadly convex, propodeal junction roundly obtuse; anterodorsal face of mesoscutum distinctly higher than anterior face of pronotum; propodeal dorsum weakly convex and gently downward sloping, rounding into steep and weakly convex posterior declivity at indistinct rounded posterior edge (Fig. 4e). In posterior view, posterior propodeal face emarginate, rounding into and undifferentiated from lateral propodeal face. In dorsal view, mesosoma obovate, notauli absent, parapsidal line indistinct (Fig. 4d). In lateral view, petiole inclined posteriorly, subtriangular, longer than high, anterior margin long, steep and weakly concave; apex indistinct from 
posterior face, forming a continuous roundly convex posteroapical face; anteroventral extension of subpetiolar process shallow and subtriangular with rounded apex (Fig. 4e). Dorsal outline of postpetiole weakly convex, differentiated from gaster by a weak but distinct cinctus (Fig. 4e).

Area between eye and antennal insertion coarsely rugulose-reticulate with interspaces punctured and weakly shiny (Fig. 4a-b); rest of head substriate-reticulate with dense punctures, and interspaces smooth and shining; area enclosed by ocelli with slightly coarser sculpture; median area immediately anterior to median ocellus and median furrow largely smooth and shining (Fig. 4a, c); clypeus coarsely rugulosereticulate with interspaces punctate and weakly shining (Fig. 4a-b). Lateral face of mesosoma largely superficially substriate-reticulate with interspaces smooth and shining (Fig. 4e). In dorsal view, mesoscutum largely superficially substriate-reticulate with interspaces smooth and shining, mesoscutal disc smooth and shining, propodeum superficially reticulate with interspaces smooth and shining (Fig. 4d). Lateral

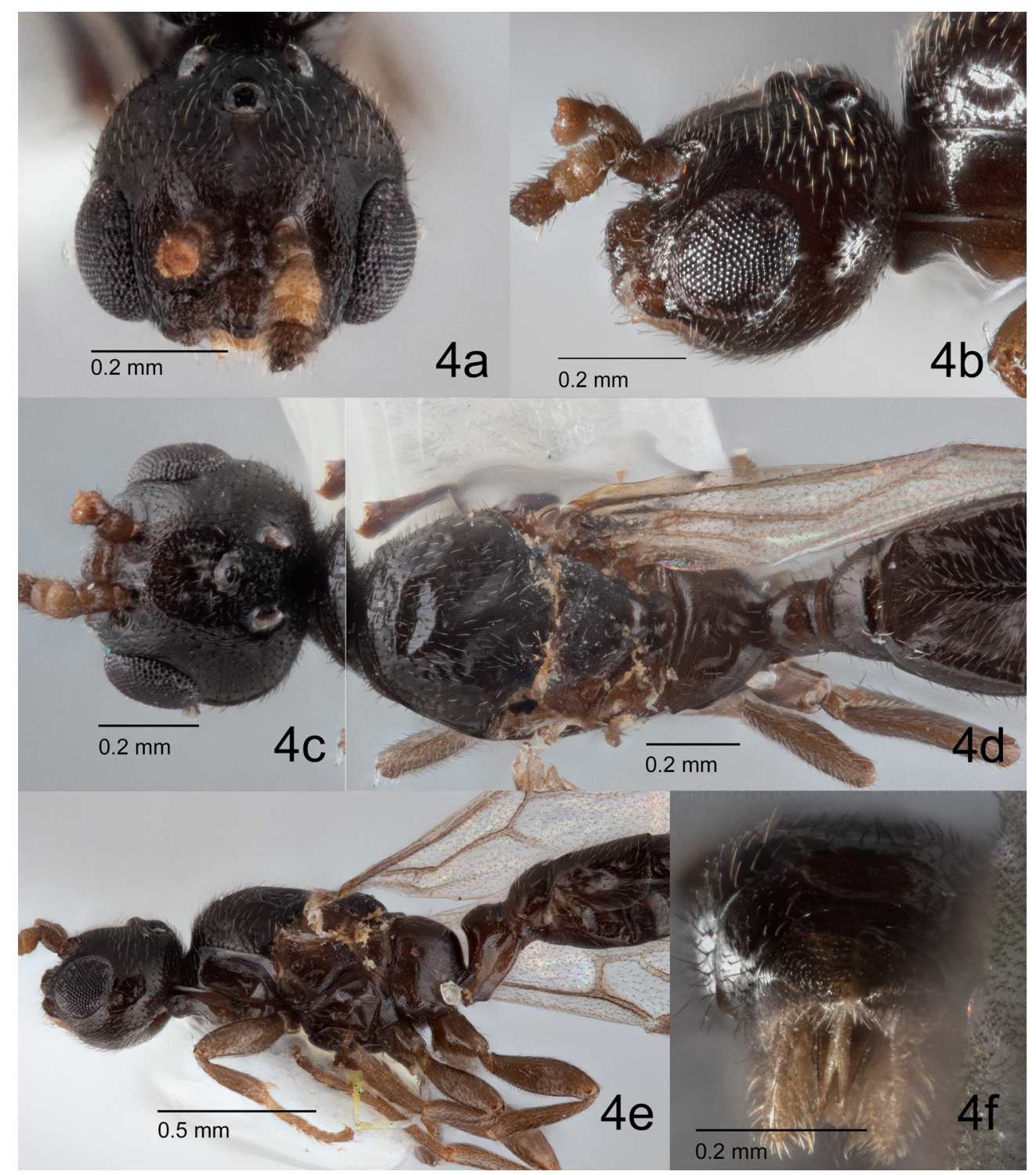

Fig. 4. Rhopalomastix impithuksai Wang \& Jaitrong sp. nov., paratype, đ (ZRC_ENT00007608). a. Head in full face view. b. Head in lateral view. c. Head in dorsal view. d. Mesosoma and waist in dorsal view. e. Body in lateral view. f. Genitalia in posterior view. 
face of petiole weakly substriate-reticulate with interspaces smooth and shining (Fig. 4e), petiolar apex entirely smooth and shining (Fig. 4d). Postpetiole weakly substriate-reticulate with interspaces smooth and shining. Gaster mostly superficially reticulate with interspaces smooth and shining.

Dorsum of head with dense short standing hairs; lateral and ventral faces with dense short decumbent and subdecumbent hairs (Fig. 4a-b). Dorsum of pronotum and mesoscutum with dense short standing hairs; posterolateral margins of propodeum with sparse short erect hairs (Fig. 4d-e). Anterior rising face of petiole without hairs, posterodorsal petiolar face including apex with short standing hairs, sparser than those on mesoscutum; dorsum of postpetiole with short erect hairs, slightly more dense than those on petiole. Gaster pilose with dense standing and subdecumbent hairs, ventral gastral hairs slightly less dense than those on gastral dorsum.

Body generally dark brown; head excluding clypeus, mesoscutum and mesoscutellar disc blackish brown; dorsum of head especially vertex, frons and area around eye with blackish undertones; antennal scape and legs uniformly greyish brown, $2^{\text {nd }}$ funicular segment yellowish brown.

\section{Distribution}

Thailand (central, Nakhon Nayok Province) (Fig. 16a).

\section{Remarks}

The worker of $R$. impithuksai Wang \& Jaitrong sp. nov. is morphologically similar to $R$. javana, but can be differentiated by the following characters: 1) condition of supraclypeal area - in R. impithuksai Wang \& Jaitrong sp. nov. the supraclypeal area is lined with dark-pigmented longitudinal carinae with posterior apex carinate and usually acute, in $R$. javana the supraclypeal area does not have such carinae, with posterior apex often indistinct; 2) hairs on anterior face of petiole - in R. impithuksai Wang \& Jaitrong sp. nov. there are few decumbent and standing hairs only present close to the apex, while in $R$. javana, there are standing hairs uniformly distributed on the entire anterior petiolar face; 3 ) upper half of outer face of mandible-finely striate in $R$. impithuksai Wang \& Jaitrong sp. nov., but smooth and shining in R. javana.

Rhopalomastix impithuksai Wang \& Jaitrong sp. nov. workers may also appear similar to the morphologically variable $R$. johorensis, but the two species can be distinguished by the aforementioned points (2) and (3). In addition, for R. impithuksai Wang \& Jaitrong sp. nov. the dorsolateral propodeal margins transition to posterior propodeal margin at bluntly rounded posterolateral corners - together with an almost flat propodeal dorsum, these give rise to a roundly obtuse posterior propodeal junction. In contrast, for $R$. johorensis, the combination of ill-defined posterolateral corners of the propodeum and a gently downward-sloping propodeal dorsum, give rise to an indistinct though broadly-rounded propodeal junction.

The large worker of $R$. impithuksai Wang \& Jaitrong sp. nov. is also morphologically similar to smaller workers of $R$. robusta Wang \& Jaitrong sp. nov., but may be differentiated based on characters described in more detail under Remarks for the latter species.

Rhopalomastix javana Wheeler, 1929

Figs 2c, 5 (worker), 6 (queen), 7 (male), 16b. Also see Wang et al. (2018b: figs 20-23) for male.

Rhopalomastix rothneyi subsp. javana Wheeler, 1929: 96, fig. 1 (w.q.m).

Rhopalomastix rothneyi subsp. javana - Chapman \& Capco 1951: 111. — Bolton 1995: 377. — Xu 1999: 131 (in key).

Rhopalomastix javana - Wang et al. 2018b: 310, figs 8-11 (raised to species, redescription). 


\section{Diagnosis}

\section{Worker}

Workers monomorphic with little size variation (types: HL $0.48-0.52$, HW $0.45-0.48$; non-types HL 0.46-0.48, HW 0.41-0.44). Head in full-face view subrectangular, longer than wide, posterior margin with a shallow median concavity (Fig. 5a). In profile, clypeus broadly and weakly convex (Fig. 5c); dorsum of head finely striate (Fig. 5a); striations on posterior half of head generally weaker and more fragmented; ventral face of head largely superficially reticulate. In dorsal view, mesosoma subrectangular with almost parallel sides and weakly concave posterior propodeal margin (Fig. 5b); posterolateral corners of propodeum bluntly angulate, slightly projecting posteriorly and distinctly, though shallowly, differentiated from inner posterior propodeal face. Propodeal junction in lateral view roundly obtuse (Fig. 5d); posterior propodeal declivitous face weakly marginate and shallowly concave (Fig. 5b, d); propodeal dorsum differentiated from posterior face by weakly marginate edge. Anterior face of petiole weakly concave (Fig. 5d), with fairly dense appressed and decumbent hairs. Head, mesosoma dorsum and gaster darker brown than rest of body; dorsum of head sometimes with blackish undertones.

\section{Queen}

Small-medium size relative to $R$. johorensis queen (non-types: HL 0.48-0.49, HW 0.44-0.45), head in full-face view subrectangular and slightly narrower anteriorly (Fig. 6a). Anterior clypeal face in profile weakly and broadly convex, sometimes almost flat (Fig. 6d). Posterior half and ventral face of head largely smooth and shiny, with feeble striations on frons and vertex. Dorsal outline of mesosoma weakly and broadly convex; propodeal junction roundly obtuse and indistinct (Fig. 6d). In dorsal view, anterior margin of pronotal disc broadly convex with roundly obtuse humeral corners; mesoscutum slightly wider than long; propodeum much wider than long; propodeal dorsum weakly convex and rounding into steep posterior declivity at indistinct rounded posterior edge; posterior propodeal face with shallow median concavity (Fig. 6c). Dorsum of mesosoma largely finely striate and shiny; lateral-most triangular area of mesoscutum immediately above parascutellar carina mostly smooth and shining (Fig. 6c); apicodorsal faces of petiole and postpetiole feebly and superficially reticulate(Fig. 6b, d), each with a central area that is smooth and shining (Fig. 6b). Body largely uniformly dark brown; head, dorsum of mesosoma and gaster slightly darker brown, tibia and tarsus yellowish brown.

\section{Male}

Small size (non-types: HL 0.39-0.41, HW 0.38-0.4). Head in full-face view sub-spherical, posterior margin broadly and strongly rounded (Wang et al. 2018b: fig. 21); in lateral view, head with dorsum gently inclined posteriorly; clypeus in profile with distinct dorsal and anterior faces separated by a roundly angulate edge, dorsal face gently sloped downwards and nearly flat, anterior face steep and very weakly convex or flat (Fig. 7a). Median ocellus subcircular, less elongate and slightly smaller than lateral ocelli; frontal lobes distinctly raised and differentiated from frontal supraclypeal area; posterior apex of supraclypeal area bluntly rounded (Wang et al. 2018b: fig. 21). In posterior view, posterior propodeal face indistinctly marginate, weakly differentiated from lateral propodeal face by indistinct rounded lateral margin. In lateral view, petiole short and subtrapezoidal, longer than high, anterior margin long, steep and weakly concave, apical dorsum weakly convex, rounding into short posterior face at indistinct rounded edge (Wang et al. 2018b: fig. 20). Funiculus of antenna uniformly light brown; legs greyish brown with joints and tarsi pale brownish yellow.

\section{Material examined}

\section{Types}

INDONESIA • lectotype (top specimen on same pin) and 2 paralectotype workers of $R$. rothneyi javana; East Java, Besoeki, Bondowoso; USNM MCZ.6.9.20783/SNM.595.31. Images generated by the same authors for a previous publication (i.e., Wang et al. 2018b), examined. 


\section{Other material}

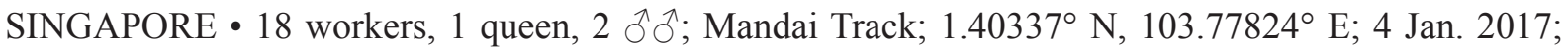
G.W. Yong leg.; colony code: GY-SG17-RhoN; ZRC_HYM000576 • 8 workers; same collection data as for preceding; THNHM $\bullet 12$ workers, 2 queens; Bukit Timah Nature Reserve; $1.35449^{\circ} \mathrm{N}, 103.78211^{\circ}$ E; 21 Jun. 2017; C. Peeters leg.; nest in bark of Campnosperma auriculatum (Blume) Hook.f.; colony code: WW-SG17-015; ZRC_HYM_0001732.

THAILAND - West Thailand - 8 workers; Kanchanaburi Province, Sai Yok District, Ban Tha Sao; $14.33389^{\circ} \mathrm{N}, 98.98^{\circ} \mathrm{E} ; 280 \mathrm{~m}$ a.s.1.; 11 Mar. 2018; W. Jaitrong leg.; mango tree; colony code: WJT1103181; THNHM -8 workers; same collection data as for preceding; GenBank: MW267108 to MW267110 (3 barcoded); ZRC_ENT00000931 - 8 workers, 1 गे; same collection data as for preceding; 11 Mar. 2018; colony code: WJT110318-2; THNHM • 8 workers, 1 ते; same collection data as for preceding; GenBank: MW267111 to MW267113 (3 barcoded); ZRC_ENT00000932. - Central Thailand • 8 workers; Saraburi Province, Phu Khae Botanical Garden; $14.67056^{\circ} \mathrm{N}, 100.88500^{\circ}$ E; 89 m a.s.1.; 18 Mar. 2018; W. Jaitrong leg.; colony code: WJT180318-2; THNHM • 8 workers; same collection data as for preceding; GenBank: MW267105 to MW267107 (3 barcoded); ZRC_ENT00000937 • 9 workers; Khao Yai National Park; 7 Jul. 2018; W. Jaitrong leg.; in bark of mango tree; colony code: WJT0707181; THNHM - 5 workers; same collection data as for preceding; GenBank: MW267096 to MW267098 (3 barcoded); ZRC_ENT00007610. - South Thailand - 8 workers, 2 queens; Trang Province, Nayong

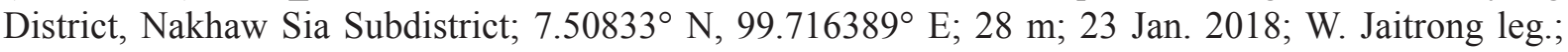
in bark of Azadirachta excelsa (Jack) Jacobs; colony code: WJT230118-1; THNHM - 8 workers, 2 queens; same collection data as for preceding; GenBank: MW267114 to MW267116 (3 barcoded); ZRC_ENT00000942. - Northern Thailand - 15 workers; Chiang Rai; 21 Oct. 2018; W. Jaitrong leg; colony code: WJT211018-34; THNHM • 8 workers; same collection data as for preceding; GenBank: MW267099 to MW267101 (3 barcoded); ZRC_ENT00007609. - Northeast Thailand • 18 workers, 1 queen; Srisa Ket Province, Phanom Dong Rak, Krabau Krabai; 13 Sep. 2018; W. Jaitrong leg.; colony code: WJT130918-17; THNHM -8 workers, 1 queen; same collection data as for preceding; GenBank: MW267117 to MW267119 (3 barcoded); ZRC_ENT00007611. - North Thailand • 8 workers; Nan Province, Nan Fa Sai Resort; 2 Dec. 2018; W. Jaitrong leg.; colony code: WJT0212187; THNHM -8 workers; same collection data as for preceding; GenBank: MW267126 to MW267128 (3 barcoded); ZRC_ENT00007869 8 workers, 2 queens; Nan Province, Puar [sic] District; 4 Dec. 2018; W. Jaitrong leg.; colony code: WJT041218-1; THNHM • 8 workers, 2 queens; same collection data as for preceding; GenBank: MW267120 to MW267122 (3 barcoded); ZRC_ENT00007870; ZRC - 13 workers, 1 queen; Nan Province, Puar [sic] District, Sirapach Waterfall; 4 Dec. 2018; W. Jaitrong leg.; colony code: WJT041218-2; THNHM • 8 workers, 1 queen; same collection data as for preceding; GenBank: MW267123 to MW267125 (3 barcoded); ZRC_ENT00007871 • 8 workers; Chiang Rai, Muang district, Huai Chom Koo subdistrict, Khun Korn waterfall; 21 Oct. 2018; W. Jaitrong and Sk. Yamane leg.; colony code: TH18-SKY-169; THNHM • 8 workers; same collection data as for preceding; GenBank: MW267102 to MW267104 (3 barcoded); ZRC_ENT00007884.

\section{Description}

\section{Worker}

Measurements. Lectotype and paralectotype workers $(\mathrm{n}=3)$, measurements from Wang et al. $(2018 \mathrm{~b})$ : EL 0.08; EW 0.04-0.06; HL 0.48-0.52; HW 0.45-0.48; ML 0.48-0.50; PronW 0.28-0.32; PtH, PtL unavailable; SL 0.18-0.20; TL 1.83-1.98; CI 92-94; REL 17-18; SI 39-42.

Non-type workers from Singapore ( $\mathrm{n}=9$ ): EL 0.08-0.1; EW 0.04-0.06; HL 0.46-0.48; HW 0.41-0.44; ML 0.50-0.54; PronW 0.28-0.32; PtH 0.15-0.18; PtL 0.17-0.20; TL 1.69-2.02; SL 0.18-0.19; CI 8993; PtHI 87-90; REL 18-23; SI 41-44. 
Non-type workers from Thailand ( $\mathrm{n}=13$ ): EL 0.08-0.12; EW 0.06-0.08; HL 0.46-0.58; HW 0.46-0.56; ML 0.56-0.76; PronW 0.30-0.36; PtH 0.17-0.23; PtL 0.19-0.25; TL 2.02- 2.88; SL 0.20-0.26; CI 9296; PtHI 89-92; REL 17-21; SI 44-48.

Workers monomorphic with little intranidal variation in size. Head in full-face view subrectangular, longer than wide, posterior margin with a broad but shallow median concavity, posterolateral corners roundly convex, lateral margins of head broadly convex and weakly converging anteriorly, anterior clypeal margin less wide than posterior margin of head, with broadly and weakly convex median section (Fig. 5a). Eye with 11-16 ommatidia for types and specimens for Singapore, 13-21 ommatidia for Thai specimens. In lateral view, outline of clypeus evenly and weakly convex, projecting slightly forward from dorsal margin of head (Fig. 5c). Mesosoma in lateral view box-shaped or subcylindrical, dorsal outline weakly and broadly convex, propodeal junction roundly obtuse, propodeal declivity steep and shallowly concave (Fig. 5d); dorsum of propodeum differentiated from posterior declivitous face by a weak but distinct marginate edge. In dorsal view, mesosoma subrectangular with lateral sides almost parallel to each other; anterior margin of pronotal disc broadly convex, sometimes angulate at its median point, humeral corners rounded and often indistinctly angulate; lateral margins of propodeum broadly convex, transitioning to and differentiated from weakly concave posterior propodeal margin at bluntly angulate

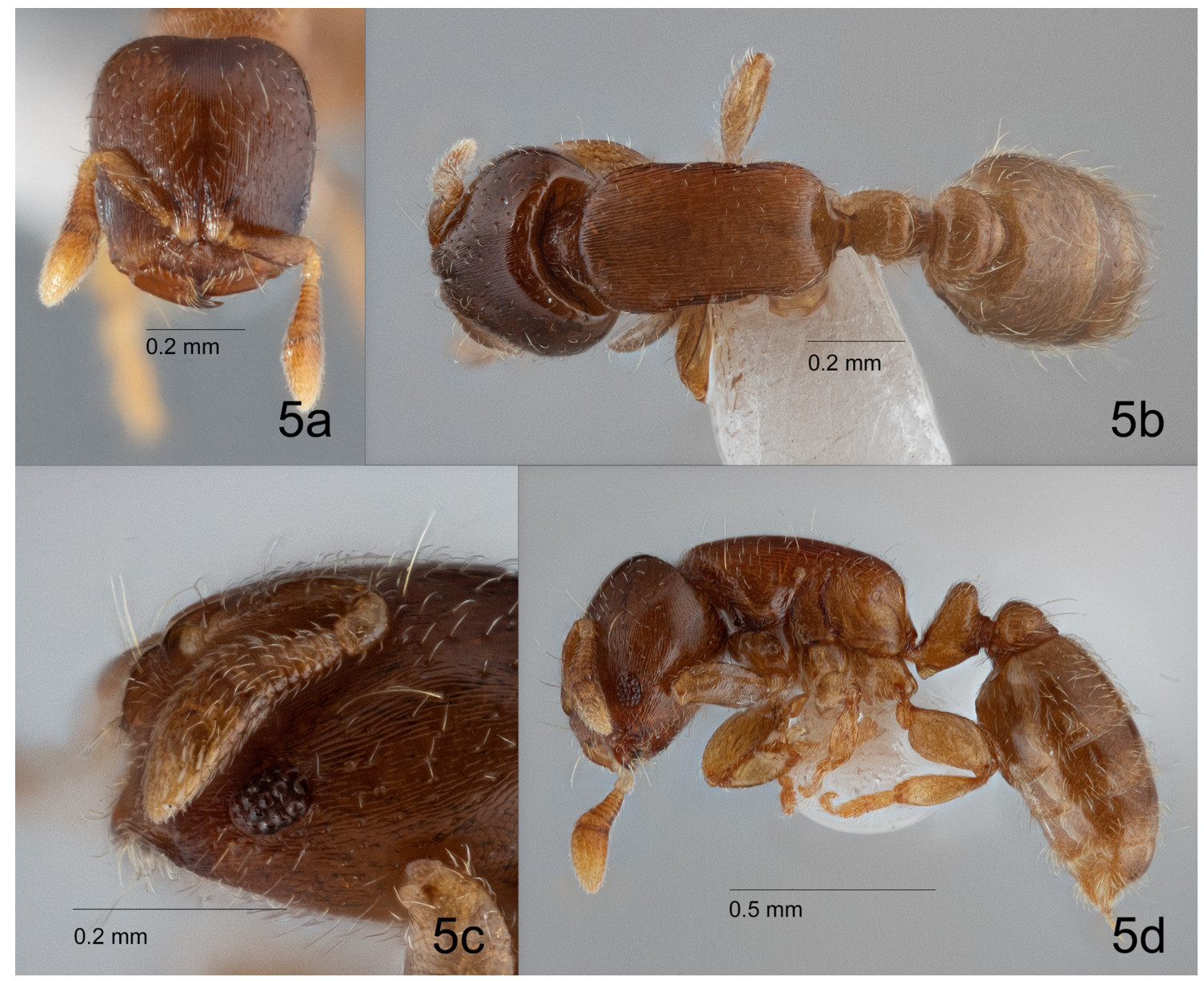

Fig. 5. Rhopalomastix javana Wheeler, 1929, non-type, worker, West Thailand (ZRC_ENT00000932). a. Head in full-face view. b. Body in dorsal view. c. Close-up of clypeus and head in profile. d. Body in lateral view. 
posterolateral corners (Fig. 5b); posterolateral corners slightly projecting posteriorly and differentiated from inner posterior propodeal face. Posterior face of propodeum weakly marginate, rounding into lateral propodeal face at indistinct angulate edge. In lateral view, petiole inclined posteriorly, slightly longer than high, distinctly narrower at apex than at base, anterior face weakly concave and longer than posterior face; apex roundly convex, apical dorsum rounding smoothly into steeper posterior face at indistinct rounded edge; petiole higher and longer than postpetiole, dorsal margin of postpetiole weakly convex and nearly flat (Fig. 5d). In dorsal view, petiole suboval, wider than long; postpetiole globular and less than twice as wide as petiole (Fig. 5b).

Dorsum and lateral face of head largely finely striate and shiny, striations on posterior half of head generally weaker and more fragmented; posterolateral corners of head feebly reticulate with interspaces smooth and shining; posteriormost strip of vertexal area just above occiput in posterodorsal view largely unsculptured or with feeble striations, mostly smooth and shiny. Ventral face of posterior half of head mostly superficially reticulate with interspaces smooth and shining. Mandible mostly smooth and shining, area immediately adjacent to masticatory margin punctate (Fig. 2c). Median clypeal face with coarse irregular striae, interspaces punctate and weakly shining (Fig. 5a), sometimes with a central longitudinal strip more weakly striate or largely smooth and shining. Lateral face of mesosoma largely striate-reticulate with interspaces smooth and shining (Fig. 5d); dorsum of mesosoma finely striate (Fig. 5b). Lateral face of petiole superficially reticulate with interspaces smooth and shining, petiolar apex finely striate and shiny. Postpetiole and gaster superficially reticulate with interspaces smooth and shining.

Dorsum of head with fairly dense though well-spaced short erect and suberect hairs interspersed with sparse long erect hairs, gena with fairly dense short appressed and decumbent hairs. Ventral face of head with fairly dense though well-spaced short suberect, decumbent and appressed hairs. Antennal funiculus mostly with fairly dense appressed and decumbent hairs. Mesosoma dorsum with sparse, scattered short standing hairs that are relatively denser closer to dorsolateral margin, paired long erect hairs sparsely present along dorsolateral margin, with one pair each flanking the anterior margin of pronotal disc, pronotum, mesonotum and propodeum. Anterior rising face of petiole with fairly dense appressed and decumbent hairs, posterior declivity with dense short erect and subdecumbent hairs; apex of petiolar node with few short erect hairs and 1-2 pairs of long erect hairs. Postpetiole with dense short erect and suberect hairs, and one pair of long erect hairs. Gaster pilose, with dense suberect and erect hairs interspersed with sparse long erect hairs.

Head, dorsum of mesosoma and gaster generally dark brown, rest of body lighter yellowish brown, dorsum of head and area around eye in some workers with blackish undertones; tips of antennae, first funicular segment and legs pale yellowish brown.

\section{Queen}

Measurements. Non-type queens $(\mathrm{n}=3)$ : EL 0.15-0.16; EW 0.09-0.10; HL 0.48-0.49; HW 0.44-0.45; ML 0.70-0.72; MsW 0.36-0.40; PtH 0.21-0.22; PtL 0.25-0.26; SL 0.18-0.20; TL 2.21-2.43; CI 92-94; PtHI 85-88; REL 33-36; SI 40-45.

Small-medium size (relative to $R$. johorensis queen). Head in full-face view subrectangular, slightly longer than wide, slightly narrower anteriorly with anterior clypeal margin distinctly less wide than posterior margin; lateral margins broadly and weakly convex, almost parallel to each other, posterolateral corners rounded, posterior margin nearly straight with a shallow median concavity (Fig. 6a). Eye very large (13-18 ommatidia in the longest axis); posteriormost point of eye located slightly below transverse midline of head in full-face view. In profile, clypeus weakly and broadly convex, sometimes almost flat (Fig. 6d). Ocelli oval in shape and generally equal in size, roughly equidistant from each other; small 
area immediately anterior to median ocellus gently depressed (Fig. 6a-b). In lateral view, mesosoma subcylindrical, more elongate than that of worker, dorsal outline weakly and broadly convex, propodeal junction roundly obtuse and indistinct (Fig. 6d). Propodeal dorsum weakly convex (Fig. 6d), rounding into steep posterior declivity at indistinct rounded posterior edge; posterior propodeal face with shallow median concavity. In dorsal view, mesosoma subcylindrical, anterior margin of pronotal disc broadly convex, humeral corners obtusely angulate, mesoscutum slightly wider than long, propodeum much wider than long, posterior margin weakly concave and indistinct, lateral margin of propodeum rounding into posterior margin at indistinct rounded edge (Fig. 6c). In lateral view, petiole inclined posteriorly, longer than high, anterior margin weakly concave and steep; petiolar node bell-shaped with bluntly rounded apex, apical face rounding smoothly into and not distinctly differentiated from posterior face, posterior face shorter and steeper than anterior face (Fig. 6d). In lateral view, postpetiole not as long as petiole, dorsal margin broadly convex (Fig. 6d). In dorsal view, petiole suboval, less than twice as wide as long; postpetiole globular, much larger and wider than petiole (Fig. 6b).

Anterior half of head dorsum largely finely striate and shiny, posterior half mostly smooth and shining with scattered punctures, frons and vertex with feeble striations; clypeus coarsely striate but with a median longitudinal strip that is smooth and shining. Ventral face of head mostly smooth and shining. Mandible mostly smooth and shining, area next to masticatory margin slightly striate-punctate. Lateral face of mesosoma weakly substriate-reticulate, interspaces smooth and shining. Dorsum of mesosoma mostly finely striate and shining, median section of pronotum superficially reticulate with interspaces smooth and shining (Fig. 6c); lateral-most triangular area of mesoscutum immediately above parascutellar carina mostly smooth and shining or more weakly striate than rest of mesoscutum (Fig. 6c); posterior

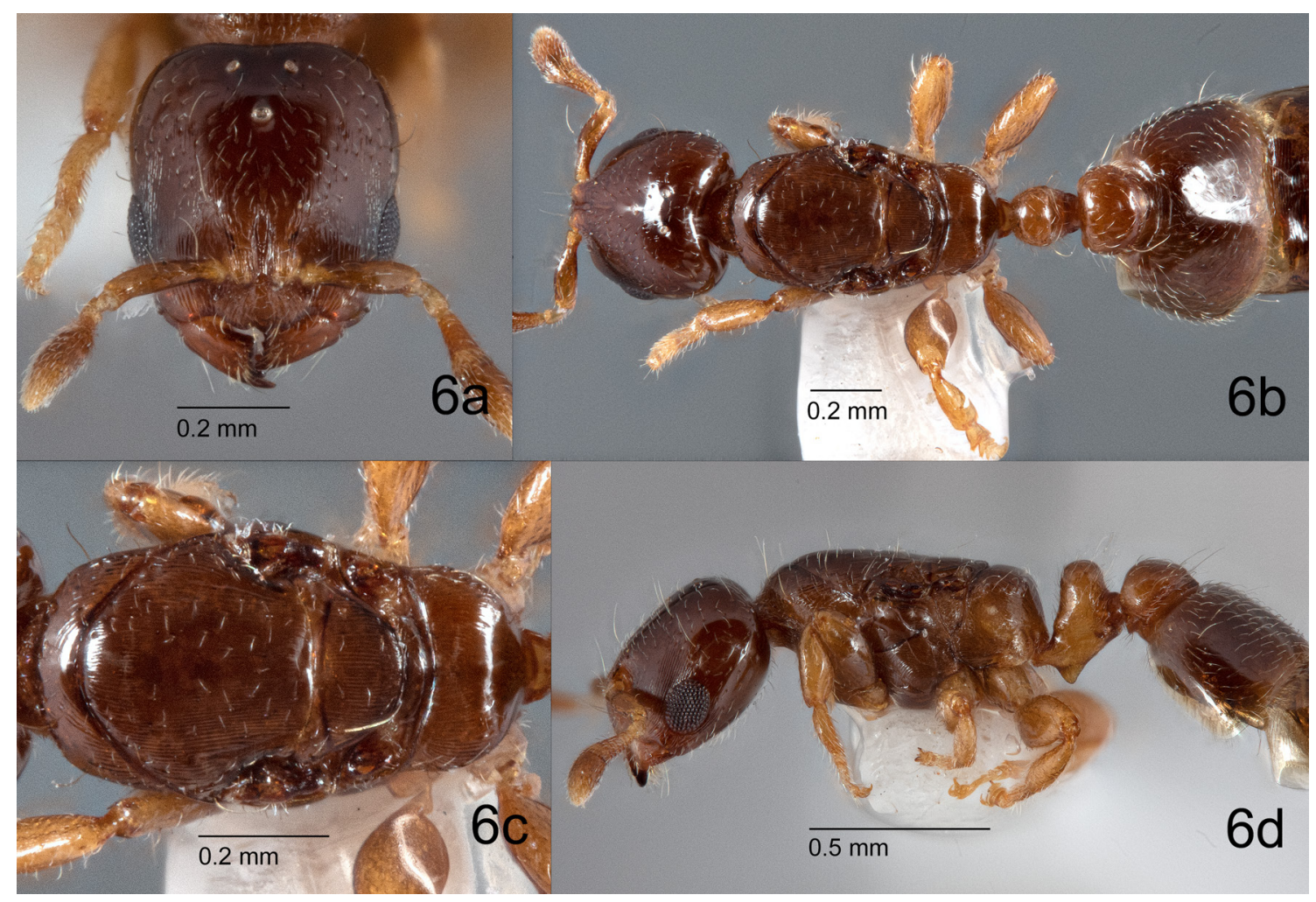

Fig. 6. Rhopalomastix javana Wheeler, 1929, non-type, queen, Northeast Thailand (THNHM-I-21751). a. Head in full-face view. b. Body in dorsal view. c. Close-up of mesosoma in dorsal view. d. Body in lateral view. 
face of propodeum mostly smooth and shining. Lateral faces of petiole and postpetiole superficially reticulate with interspaces smooth and shining; apicodorsal faces of petiole and postpetiole feebly and superficially reticulate with a central area that is smooth and shining. Gaster largely smooth and shining, area near anterior margin of tergite superficially reticulate.

Dorsum of head with fairly dense short suberect and decumbent hairs, interspersed with very sparse long erect hairs, including one pair on median section of clypeus; lateral and ventral faces with fairly dense short appressed and decumbent hairs. Antennal scape with uniform short standing hairs and a long erect hair on basal and median points respectively. Dorsum of mesosoma with sparse short standing hairs (Fig. 6c); paired long erect hairs sparsely present along dorsolateral margin, with one pair each flanking the anterior margin of pronotal disc, pronotum, mesoscutum, and propodeum; mesoscutum and mesoscutal disc each one pair of long erect hairs. Anterior face of petiole with dense short appressed and/or decumbent hairs, posterior face with dense short erect hairs, apex with sparse short erect hairs, 1-2 pairs of long erect hairs. Postpetiole with dense short decumbent or standing hairs and two pairs of long erect hairs. Gaster pilose, with dense short appressed and/or decumbent hairs, scattered short standing hairs, and long erect hairs sparsely distributed along posterior margins of tergites.

Body generally uniform dark brown; head, dorsum of mesosoma and gaster slightly darker brown; tibia and tarsus paler and yellowish brown.

\section{Male}

Measurements. Thai male specimens unavailable. Non-type males from Singapore $(\mathrm{n}=2)$ : EL 0.18-0.20; EW 0.14; HL 0.39-0.41; HW 0.38-0.40; ML 0.66-0.70; MsW 0.40-0.44; PtH 0.17-0.18; PtL 0.210.22; SL 0.06; TL 1.98-1.99; CI 97-98; REL 45-53; SI 15-16.

Small size (relative to $R$. johorensis male). Head in full-face view sub-spherical, posterior margin broadly and strongly rounded (Wang et al. 2018b: fig. 21). Eye very large (20-25 ommatidia in the longest axis); posteriormost point of eye only slightly exceeding transverse midline of head, outline of eye bulging from lateral margin of head by a little (Wang et al. 2018b: fig. 21). Median ocellus subcircular, less elongate and slightly smaller than lateral ocelli; small area of head dorsum immediately anterior to median ocellus weakly depressed, continuous with weak median furrow separating frontal lobes; frontal lobes strongly raised and differentiated from supraclypeal area (Wang et al. 2018b: fig. 21). Posterior apex of supraclypeal area bluntly rounded. In lateral view, head broad-ovate with dorsum gently inclined posteriorly, posterodorsal outline broadly rounded with differentiation between dorsal and posterior faces indistinct, posterior margin rounding into broadly convex ventral margin forming a continuous curve with no distinct posteroventral angle (Fig. 7a). In the same view, clypeus strongly projecting from dorsum of head, with distinct dorsal and anterior faces separated by a roundly angulate edge, dorsal face gently sloped downwards and nearly flat, anterior face steep and very weakly convex or flat (Fig. 7a).

In lateral view, mesosoma sub-oblong, dorsal margin almost completely straight or very weakly and broadly convex, propodeal junction roundly obtuse; anterior face of pronotum about as high as anterodorsal face of mesoscutum (Wang et al. 2018b: fig. 20); propodeal dorsum weakly convex, rounding into steep and short posterior declivity at indistinct rounded posterior edge. In posterior view, posterior propodeal face indistinctly marginate, weakly differentiated from lateral propodeal face by indistinct rounded lateral margin. In dorsal view, mesosoma obovate, notauli absent, parapsidal line weakly present (Wang et al. 2018b: figs 22-23). In lateral view, petiole inclined posteriorly, short and subtrapezoidal, longer than high, anterior margin long, steep and weakly concave, apical dorsum weakly convex, rounding into short posterior face at indistinct rounded edge; anteroventral extension of subpetiolar process shallow and subtriangular, longer at base than apex (Wang et al. 2018b: fig. 20). 
Dorsal outline of postpetiole weakly convex, differentiated from gaster by a weak but distinct cinctus (Wang et al. 2018b: fig. 20).

Head mostly superficially substriate-reticulate with interspaces smooth and shining, and scattered punctures (Wang et al. 2018b: fig. 21); area of head dorsum enclosed by and around ocelli rugulose and weakly shining; median area immediately anterior to median ocellus largely smooth and shining; frontal lobes coarsely rugulose-reticulate and weakly shining; clypeus coarsely rugulose with interspaces punctate and weakly shining. Lateral face of mesosoma weakly striate-reticulate with interspaces smooth and shining (Wang et al. 2018b: fig. 20). In dorsal view, mesoscutum largely weakly substriate with interspaces smooth and shining, lateralmost area of mesoscutum in between parapsidal line and parascutellar carina unsculptured, largely smooth and shining; mesoscutal disc largely smooth and shining with feeble superficial striations; propodeum superficially reticulate with interspaces smooth and shining (Wang et al. 2018b: fig. 23). Petiole, postpetiole mostly reticulate with interspaces smooth and shining, apices smooth and shining.

Dorsum of head with dense short standing hairs; lateral, ventral and posterior faces with dense short decumbent and appressed hairs (Fig. 7a). Dorsum of pronotum and mesoscutum with fairly dense short standing hairs; posterolateral margins of propodeum with sparse short erect hairs. Anterior rising face of petiole with few or no hairs, apicodorsal and posterior petiolar faces with short standing hairs; dorsum of postpetiole with short standing hairs. Gaster pilose with dense standing, decumbent and subdecumbent hairs.

Body generally dark greyish-brown; head, dorsum of mesosoma and gaster dark blackish-brown; funiculus of antenna uniformly light brown; legs greyish brown with joints and tarsi pale brownish yellow.

\section{Distribution and binomics}

Indonesia (Java), Singapore, Thailand (Fig. 16b). As with R. johorensis, colonies can be usually found in (but not limited to) bark of fruit trees such as mango, located close to human dwellings. Populations appear widespread, but more sparsely distributed and less commonplace than those of $R$. johorensis this could merely be an artefact of incomplete sampling.

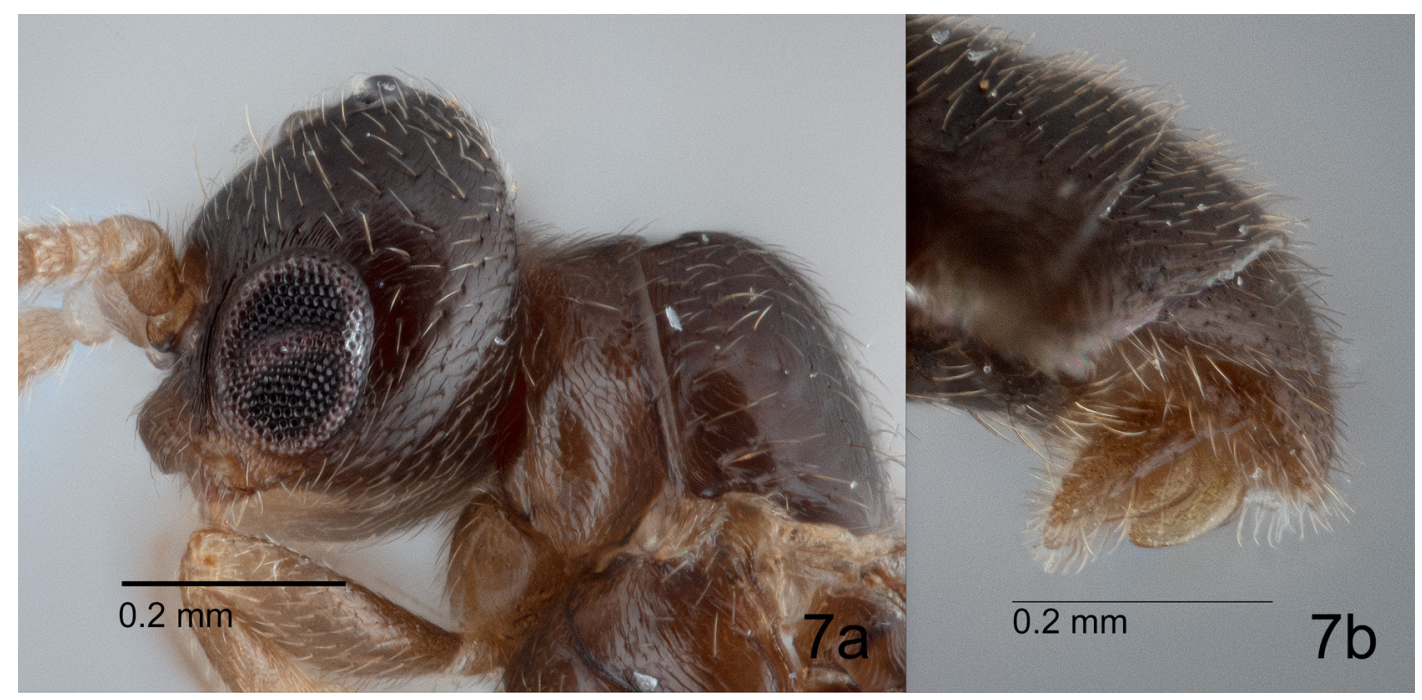

Fig. 7. Rhopalomastix javana Wheeler, 1929, non-type, $\widehat{\jmath}$, Singapore (ZRC_HYM_0000576). a. Head and clypeus in profile. b. Close-up of genitalia in profile. 


\section{Remarks}

Thai populations of $R$. javana appear to be generally larger in size compared to populations from Indonesia (Java) and Singapore (Java and Singapore TL 1.83-2.02; Thailand TL 2.02-2.88). In terms of variation in morphological measurement metrics, Thai populations also seem much more variable relative to the other two countries (e.g., Thai HL 0.46-0.58; Java HL 0.48-0.52; Singapore HL 0.460.48 ), however, this might have arisen from bias in geographic range of sampling. For Thai $R$. javana, measurements were taken from 13 individuals from seven colonies distributed across Central, North and West Thailand, whereas for Java and Singapore, mostly individuals from 1-2 colonies were measured. A combination of measurements from different countries may be a more accurate representation of actual variation within the species.

Thai colonies that could be morphologically identified as $R$. javana were extremely variable in terms of COI (313 bp), with internidal divergences spread across multiple different values from $0.0-4.5 \%$ (Fig. 1). While sequence divergence between different species existing in sympatry can be as low as $3.8 \%$ (average uncorrected $p$ distance) in ants, especially for recently diverged species (Wang et al. 2018a), higher percentage distances $(>4 \%)$ between allopatric populations do not necessarily indicate different species. In the latter case, clustering based on barcodes may split allopatric or geographically distant populations of the same species, belonging to older lineages that diverged genetically back in time (Meier et al. 2008). Thus, in light of the two aforementioned possibilities, inferring species boundaries from the clustering pattern of Thai $R$. javana specimens is complicated. If we define allopatric colonies as those coming from different parts (i.e., North, South, East, West, Central) of Thailand, then possibly the split clusters observed (Fig. 1) mostly represent different lineages of the same species $R$. javana. However, $R$. javana colonies considered 'sympatric' from North and Central Thailand respectively diverged at $4.5 \%$ (Fig. 1), suggesting (but not ascertaining) possible newly diverged species. Taking into account the broad inter- and intranidal variation shown amongst $R$. javana workers, we were unable to observe any striking morphological differences between specimens from the genetically divergent clusters. Given the lack of more convincing morphological or molecular evidence supporting further species delimitation in this study, we tentatively treat these colonies as conspecific. The possibility remains for this current treatment to be revised in future, when gene flow between $R$. javana populations are more thoroughly investigated, and the relevant data from other molecular markers are made available.

This species is most similar to R. impithuksai Wang \& Jaitrong sp. nov. and R. johorensis, but can be differentiated by characters described in detail under the Remarks for each of the latter two species.

Rhopalomastix johorensis Wheeler, 1929

Figs 2d, 8 (worker), 9 (queen), 10 (male), 16b

Rhopalomastix rothneyi subsp. johorensis Wheeler, 1929: 96 (w).

Rhopalomastix janeti Donisthorpe, 1936: 55 (w.q.m).

Rhopalomastix rothneyi johorensis - Chapman \& Capco 1951: 111 (misspelt as "R. rothneyi jahorensis").

— Bolton 1995: 377. — Xu 1999: 131 (in key). — Wang et al. 2018b: 316, figs 24-27.

Rhopalomastix johorensis - Wang et al. 2018b: 316 (raised to species).

\section{Diagnosis}

\section{Worker}

Monomorphic workers with broad size variation (types and non-types: HL 0.44-0.58; HW 0.42-0.56). Head of large worker slightly more rectangular than small worker; posterior margin with shallow median depression (Fig. 8a). In profile, anterior clypeal face either weakly and broadly convex or almost 
entirely flat or straight (Fig. 8c); dorsum and lateral face of head finely striate; ventral surface of head reticulate with smooth and shiny interspaces; outer surface of femora and first gastral tergite superficially reticulate with smooth and shiny interspaces. Dorsal outline of mesosoma weakly and broadly convex with propodeal dorsum gently downward-sloping (Fig. 8d). Anterior margin of pronotal disc broadly convex, humeral corners roundly obtuse or indistinct (Fig. 8b). Lateral margins of propodeum in dorsal view broadly convex and posteriorly converging, posterior margin very weak, posterolateral corners usually indistinct, sometimes bluntly and shallowly rounded (Fig. 8b); propodeal junction in lateral view indistinct and broadly rounded (Fig. 8d); posterior propodeal face incompletely marginate with obscure dorsal edge, and almost flat or shallowly concave in upper half. Head and dorsum of mesosoma darker brown compared to rest of yellowish-brown body; legs and tips of antennae slightly paler in tone. Large workers generally darker brown compared to small workers.

\section{Queen}

Relatively large size (non-types: HL $0.54-0.56$, HW $0.51-0.53$ ), head not much more rectangular compared to large worker (Fig. 9a). In profile, clypeus very weakly convex and almost entirely flat (Fig. 9d). In dorsal view, anterior margin of pronotal disc strongly convex and sometimes angulate at median point, humeral corners obtusely angulate (Fig. 9b). Propodeal outline in lateral view roundly convex (Fig. 9d); propodeal dorsum smoothly rounds into steep posterior face at indistinct rounded posterior edge; posterior face of propodeum weakly convex; in dorsal view propodeum generally subsemicircular in shape (Fig. 9c). Anterior half of head finely striate; posterior half mostly smooth and shining with scattered punctures. Entire dorsum of mesosoma finely striate and shiny (Fig. 9c); lateral face of mesosoma striate-reticulate with interspaces smooth and shining (Fig. 9d). Overall darker blackish brown as compared to worker, with identical tones.

\section{Male}

Relatively large size (non-types: HL 0.44 , HW 0.4-0.42). Head in full-face view broad ovate and somewhat narrower posteriorly (Fig. 10a), in lateral view narrowed posteroapically with steep dorsum (Fig. 10b). Clypeus in profile projected strongly from dorsal margin of head, short dorsal margin separated from longer anterior margin at roundly angulate edge, anterior clypeal face weakly convex or almost flat (Fig. 10b). Median ocellus subcircular, lateral ocelli suboval (Fig. 10a). Petiole subtriangular with bluntly rounded apex, apex rounding into posterior face forming a continuous convex slope (Fig. 10e). Anterior half of head dorsum rugulose-reticulate, interspaces punctured and weakly shining; punctures on posterior face of head more dense than rest of head; area enclosed by ocelli rugulose-punctate and weakly shining. Mesosoma mostly superficially substriate-reticulate with smooth and shining interspaces. Body colour generally dark grey-brown, head blackish; antennae and joints of legs pale light brown.

\section{Material examined}

\section{Types}

SINGAPORE - lectotype (on top of a pin) and 2 paralectotype workers of $R$. rothneyi subsp. johorensis; Overbeck leg.; MCZ_ENT00023066. Images generated by the same authors for previous publication (i.e., Wang et al. 2018b), examined.

\section{Other material}

INDONESIA - 2 workers; West Java, Bogor, Kebun Raya; 6 Dec. 1995; F. Ito leg.; colony code: FI95462; ZRC_ENT00007886 • 3 workers, 1 queen, 1 đ̊; West Java, ITB Campus; ca $700 \mathrm{~m}$ a.s.1.; 28 Dec. 2002; Sk. Yamane leg.; under bark of large tree; colony code: JV02/03-SKY-16; ZRC_ENT00000745.

SINGAPORE $\bullet 7$ workers, 1 queen; Mandai Road; $1.41385^{\circ}$ N, $103.80481^{\circ}$ E; 29 Nov. 2016; G.W. Yong and B. Ho leg.; nest behind bark of living tree; colony code: GY-SG16-RhoJ; ZRC_HYM_0000509 • 12 workers, 1 queen; Hougang Avenue 3; $1.34769^{\circ}$ N, $103.88838^{\circ}$ E; 12 Dec. 2016; G.W. Yong and 
S.X. Chui leg.; mango tree; colony code: GY-SG16-RhoL; ZRC_HYM_0000511 • 12 workers, 1 queen; Pulau Tekukor; $1.23081^{\circ}$ N, 103.83774 E; 15 Jan. 2017; G.W. Yong leg.; GY-SG17-RhoP; ZRC_ HYM_0000578 • 12 workers; Mandai Road; $1.41318^{\circ}$ N, $103.79405^{\circ}$ E; 21 Nov. 2016; G.W. Yong and S.X. Chui leg.; nest behind bark of Aquilaria malaccensis Lam.; colony code: GY-SG16-RhoH; ZRC_HYM_0000291 - 6 workers, 1 \% ; same collection data as for preceding; nest behind bark of Macaranga gigantea (Rchb.f. \& Zoll.) Müll.Arg.; colony code: GY-SG16-RhoI; ZRC_HYM_0000292 • 12 workers; Pulau Tekukor; $1.23081^{\circ} \mathrm{N}, 103.83774^{\circ} \mathrm{E}$; 9 Dec. 2016; G.W. Yong and Y.G. Tan leg.; mango tree; colony code: GY-SG16-RhoK; ZRC_HYM_0000510 • 12 workers; Mandai Road; $1.41333^{\circ} \mathrm{N}$, $103.79839^{\circ}$ E; 13 Jan. 2017; G.W. Yong leg.; colony code: GY-SG17-RhoO; ZRC_HYM_0000577• 12 workers, 1 '万; same collection datas as for preceding; 18 Nov. 2016; G.W. Yong and S.X. Chui leg.; nest behind bark of Campnosperma auriculatum; colony code: GY-SG16-RhoF; ZRC_HYM_0000289 - 12 workers, 2 queens; Bukit Timah Nature Reserve; $1.35127^{\circ}$ N, $103.78161^{\circ}$ E; 1 Nov. 2017; W. Wang leg.; nest in bark of Artocarpus integer (Thunb.) Merr., alias cempedak; colony code: WW-SG17-022; ZRC_HYM_0001794 • 8 workers, 2 queens, 1 ô; Kay Siang Road; 3 Jan. 1992; D.H. Murphy leg.; on bark of Mangifera indica; ZRC_HYM_0000592.

THAILAND - Central Thailand - 8 workers; Pathum Thani Province, Khlong Luang District, Khlong 5; $14.18083^{\circ} \mathrm{N}, 100.71^{\circ} \mathrm{E} ; 3 \mathrm{~m}$ a.s.l.; 3 Mar. 2018; W. Jaitrong leg.; mango tree; colony code: WJT030318-1; THNHM - 8 workers; same collection data as for preceding; GenBank: MW267085 to MW267087 (3 barcoded); ZRC_ENT00000925 - 8 workers; same collection data as for preceding; colony code: WJT130218-1; THNHM - 8 workers; same collection data as for preceding; GenBank: MW267082 to MW267084 (3 barcoded); ZRC_ENT00000930 - 8 workers; Pathum Thani Province, Khlong Luang District, Khlong 3; $14.17528^{\circ} \mathrm{N}, 100.66333^{\circ} \mathrm{E}$; $10 \mathrm{~m}$ a.s.1.; 9 May. 2018; W. Jaitrong leg.; mango tree; colony code: WJT090518-1; THNHM • 8 workers; same collection data as for preceding; GenBank: MW267088 to MW267090 (3 barcoded); ZRC_ENT00007612 • 8 workers; same collection data as for preceding; colony code: WJT090518-2; THNHM -8 workers; same collection data as for preceding; GenBank: MW267091 to MW267093 (3 barcoded); ZRC_ENT00007613 • 8 workers; Ang Thong Province, Chaiyo District, Lakfa Subdistrict; $14.68278^{\circ} \mathrm{N}, 100.46444^{\circ} \mathrm{E} ; 14 \mathrm{~m}$ a.s.1.; $7 \mathrm{Apr}$. 2018; W. Jaitrong leg.; colony code: WJT070418-2; THNHM • 8 workers; same collection data as for preceding; GenBank: MW267079 to MW267081 (3 barcoded); ZRC_ENT00000926 • 8 workers; same collection data as for preceding; colony code: WJT070418-3; THNHM $\bullet 8$ workers; same collection data as for preceding; ZRC_ENT00000927 • 8 workers; same collection data as for preceding; colony code: WJT070418-4; THNHM - 8 workers; same collection data as for preceding; GenBank: MW267076 to MW267078 (3 barcoded); ZRC_ENT00000928 • 8 workers; same collection data as for preceding; colony code: WJT070418-5; THNHM -8 workers; same collection data as for preceding; GenBank: MW267073 to MW267075 (3 barcoded); ZRC_ENT00000929 • 8 workers; Saraburi Province, Phu Kae Botanical Garden; $14.67056^{\circ} \mathrm{N}, 100.885^{\circ} \mathrm{E} ; 89 \mathrm{~m}$ a.s.1.; 18 Mar. 2018; W. Jaitrong leg.; colony code: WJT180318-1; THNHM - 8 workers; same collection data as for preceding; GenBank: MW267094 to MW267095 (2 barcoded); ZRC_ENT00000936 - 8 workers; Saraburi Province, Kang Koi District, Ban Chaom, nr. Ched Kod waterfall; $14.47111^{\circ} \mathrm{N}, 101.16111^{\circ}$ E; 8 Jan. 2018; W. Jaitrong leg.; colony code: WJT080118-3; THNHM • 8 workers; Nakhon Nayok Province, Ban Na District; 11 May 2018; W. Jaitrong leg.; from mango tree; colony code: WJT110518-1; THNHM • 8 workers; Nakhon Prathom Province, Kam Pang Sean District, Kasetsart Campus; 28 Sep. 2018; W. Jaitrong leg.; colony code: WJT280918-1; THNHM • 8 workers; same collection data as for preceding; GenBank: MW267070 to MW267072 (3 barcoded); ZRC_ENT00007601 • 8 workers, 2 queens, 3 o $\partial^{7}$; Pathum Thani Province, Khlong 6, Khlong luang, Ratchamangkala University; $14.0384^{\circ} \mathrm{N}, 101.7272^{\circ} \mathrm{E} ; 850 \mathrm{~m}$ a.s.1.; 16 Nov. 2019; W. Wang leg.; yard next to university building, nest in bark of Mangifera indica L. (mango) tree; colony code: WW-THL19-001; THNHM $\bullet 8$ workers, 2 queens, 3 ôं; same collection data as for preceding; ZRC_ENT00013956. - East Thailand - 8 workers; Chanthaburi Province, Tha Mai District, Burapecholathid Road; $12.57083^{\circ} \mathrm{N}, 101.91611^{\circ} \mathrm{E} ; 5 \mathrm{~m}$ a.s.1.; 17 Mar. 2018; W. Jaitrong leg.; colony 
code: WJT170318-1; THNHM • 8 workers; same collection data as for preceding; GenBank: MW267058 to MW267060 (3 barcoded); ZRC_ENT00000933 • 8 workers, 1 queen; Chanthaburi Province, Tha Mai District, Khlong Khud Subdistrict; $12.57167^{\circ}$ N, 101.93111 ${ }^{\circ}$ E; 18 m a.s.1.; 17 Mar. 2018; W. Jaitrong leg.; colony code: WJT170318-2; THNHM • 8 workers, 1 queen; same collection data as for preceding; GenBank: MW267055 to MW267057 (3 barcoded); ZRC_ENT00000934 • 8 workers; same collection data as for preceding; colony code: WJT170318-3; THNHM - 8 workers; same collection data as for preceding; GenBank: MW267052 to MW267054 (3 barcoded); ZRC_ENT00000935. - South Thailand - 8 workers; Trang Province, Nayong District, Ban Nakhaw Sia; 7.50833 N, 99.71639 E E; 24 Jan. 2018; W. Jaitrong leg.; under bark of Azadirachia excelsa; colony code: WJT240118-2; THNHM - 8 workers; same collection data as for preceding; GenBank: MW267061 to MW267063 (3 barcoded); ZRC ENT00000940 • 8 workers; same collection data as for preceding; colony code: WJT240118-3; THNHM - 8 workers, 1 queen; same collection data as for preceding; colony code: WJT240118-4; THNHM

- 8 workers, 1 queen; same collection data as for preceding; GenBank: MW267064 to MW267066 (3 barcoded); ZRC_ENT00000941 • 10 workers, 2 queens; Narathiwat Province, Sunuaikolok; 30 Jun. 2018; W. Jaitrong leg.; nest in bark of mango tree; colony code: WJT300618-11; THNHM • 8 workers, 2 queens; same collection data as for preceding; GenBank: MW267067 to MW267069 (3 barcoded); ZRC_ENT00007607.

\section{Description}

\section{Worker}

Measurements. Non-type workers $(\mathrm{n}=18)$ : EL 0.08-0.12; EW 0.05-0.08; HL 0.44-0.58; HW 0.420.56; ML 0.52-0.74; PronW 0.28-0.42; PtH 0.13-0.20; PtL 0.15-0.23; SL 0.17-0.22; TL 1.84-2.43; CI 92-97; PtHI 83-90; REL 16-21; SI 37-45.

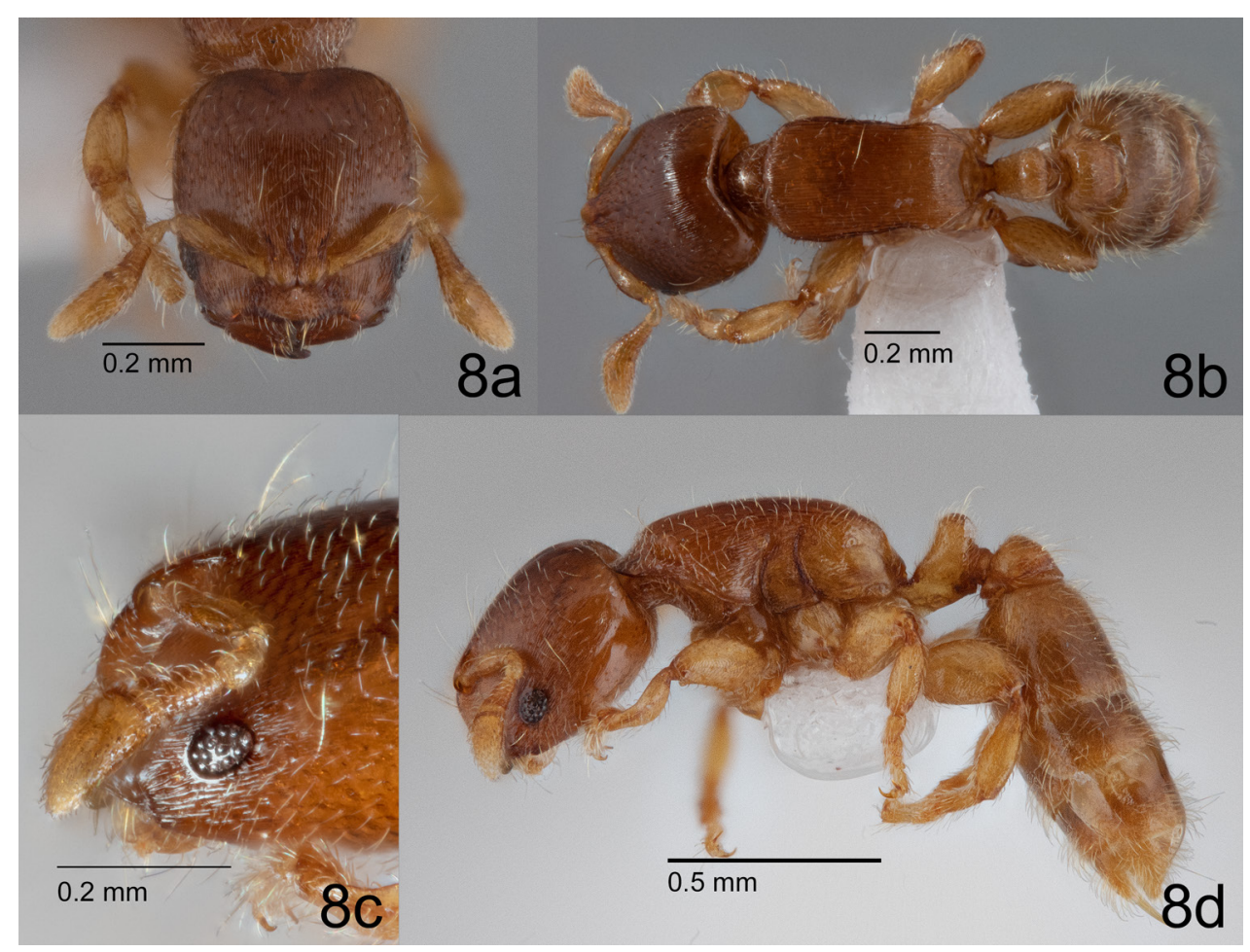

Fig. 8. Rhopalomastix johorensis Wheeler, 1929, non-type, worker, Bangkok, Thailand (ZRC ENT00007874.1). a. Head in full-face view. b. Body in dorsal view. c. Closeup of clypeus in profile. d. Body in lateral view. 
Workers monomorphic with broad intranidal size variation. Head in full-face view sub-rectangular, longer than wide (only slightly in small worker), slightly less wide anteriorly in large workers; median furrow between frontal lobes short, not extending past transverse midline of head; posterolateral corners smoothly rounded, lateral margins broadly and weakly convex, almost parallel to each other, posterior margin with shallow median depression (Fig. 8a). Eye relatively large, with 17-29 ommatidia (10-15 in smaller workers). In profile, clypeus projecting forward slightly from dorsal margin of head, anterior clypeal face largely flat or weakly convex (Fig. 8c). Antennal scape short and thin, extending slightly beyond midlength of head when lain backwards. Mesosoma in lateral view box-shaped, dorsal outline weakly and broadly convex with propodeal dorsum gently downward-sloping, propodeal junction indistinct and broadly rounded (Fig. 8d); propodeal dorsum rounding into posterior declivitous face at indistinct rounded edge, posterior face of propodeum weakly and incompletely marginate with obscure dorsal edge, almost flat or sometimes shallowly concave in upper half. In dorsal view, mesosoma subcylindrical, anterior margin of pronotal disc broadly convex, sometimes slightly angulate at median point in large worker, humeral corners roundly obtuse and often indistinct, dorsolateral margins almost straight and nearly parallel to each other, not much narrower posteriorly than anteriorly, lateral sides of propodeum broadly convex and posteriorly convergent, posterior propodeal margin very weak and indistinct (Fig. 8b); posterolateral corners of propodeum usually indistinct and ill-defined, sometimes bluntly and shallowly rounded, indistinctly differentiated from inner posterior propodeal face. In lateral view, petiole inclined posteriorly and bell-shaped, slightly longer than high, anterior face steep and weakly concave, apex rounded, about as high as postpetiole, posterior face less high than anterior face and slightly steeper; dorsal outline of postpetiole weakly convex (Fig. 8d). In lateral view, anteroventral extension of subpetiolar process short, subtriangular, variable in size across individuals, posterior margin of subpetiolar process usually almost straight or weakly and broadly convex (Fig. 8d). In dorsal view, both petiole and postpetiole oblong or elliptical, both wider than long; postpetiole larger and wider than petiole (Fig. 8b).

Dorsum and lateral face of head finely striate and shiny; median clypeal face coarsely striate and weakly shining (Fig. 8a); ventral face of head striate-reticulate with interspaces smooth and shining. Mandible mostly smooth and shining but densely punctate near masticatory margin, basally with weak striations (Fig. 2d). Lateral face of mesosoma substriate-reticulate and shiny with interspaces punctate and weakly shining, coarser sculpture near dorsal margin (Fig. 8d). Petiole and postpetiole largely superficially reticulate with interspaces smooth and shining, apicodorsal face of petiole sometimes weakly striate. Gaster largely superficially reticulate with interspaces smooth and shining, and scattered punctures.

Head and antenna with abundant short standing and suberect hairs; sparse long erect hairs mainly on clypeus, antennal scape and faint outline of antennal scrobe depression. Mesosomal dorsum with abundant but scattered short standing hairs (Fig. 8b, d), paired long erect hairs present along dorsolateral margin, with one pair each flanking anterior margin of pronotal disc, pronotum, mesonotum and propodeum. Anterior petiolar face with dense standing and/or appressed hairs; apical faces of petiole, postpetiole each with dense short standing hairs of nearly uniform length, and 1-2 pairs of long erect hairs. Dorsal and ventral faces of gaster with abundant and dense erect, suberect and decumbent short hairs, these hairs much denser and longer than those on head and mesosoma, interspersed with sparse long erect hairs.

Small workers generally uniform pale yellowish-brown in colour; head and mesosoma of slightly darker tone, especially the dorsal surface of the mesonotum. Large workers darker brown or more reddish brown compared to small workers, tonal patterns identical.

\section{Queen}

Measurements. Non-type queens ( $\mathrm{n}=7$ ): EL 0.16-0.18; EW 0.12-0.13; HL 0.54-0.56; HW 0.51-0.53; ML 0.8-0.88; MsW 0.42-0.46; PtH 0.22-0.25; PtL 0.25-0.29; SL 0.2-0.22; TL 2.53-3.1; CI 94-96; PtHI 84-89; REL 31-35; SI 38-43. 
Relatively large compared to worker. Head in full-face view subrectangular, posterolateral corners roundly angulate, posterior margin nearly straight with a weak median depression (Fig. 9a). Eye very large (13-18 ommatidia in the longest axis); posteriormost point of eye located just below transverse midline of head in full-face view (Fig. 9a). In profile, clypeus projected slightly forward from dorsal margin of head, very weakly convex or almost straight and flat (Fig. 9d). Scape short, barely surpassing posteriormost point of eye when lain back on head. Ocelli small and suboval, generally similar in size and equidistant from each other (Fig. 9a-b). In lateral view, mesosoma subcylindrical, more elongate than large worker, dorsal outline broadly and weakly convex, propodeal outline roundly convex; sulci on lateral face of mesosoma distinct (Fig. 9d). Propodeal dorsum not distinctly differentiated from and smoothly rounding into steep posterior face at indistinct rounded posterior edge; posterior face of propodeum weakly convex. In dorsal view, mesosoma subcylindrical, anterior margin of pronotal disc strongly convex and sometimes angulate at median point, humeral corners obtusely angulate, mesoscutum almost as wide as long; sides of propodeum broadly convex and posteriorly converging, posterior propodeal margin indistinct, entire propodeum generally sub-semicircular in shape (Fig. 9bc). In lateral view, petiole subtrapezoidal, slightly inclined posteriorly, anterior petiolar face weakly concave and steep; apex of petiolar node rounded and slightly higher than postpetiole; posterior petiolar face less high and steeper than anterior face (Fig. 9d). In dorsal view, petiole suboval, less than twice as wide as long; postpetiole globular, broader and larger than petiole (Fig. 9b).

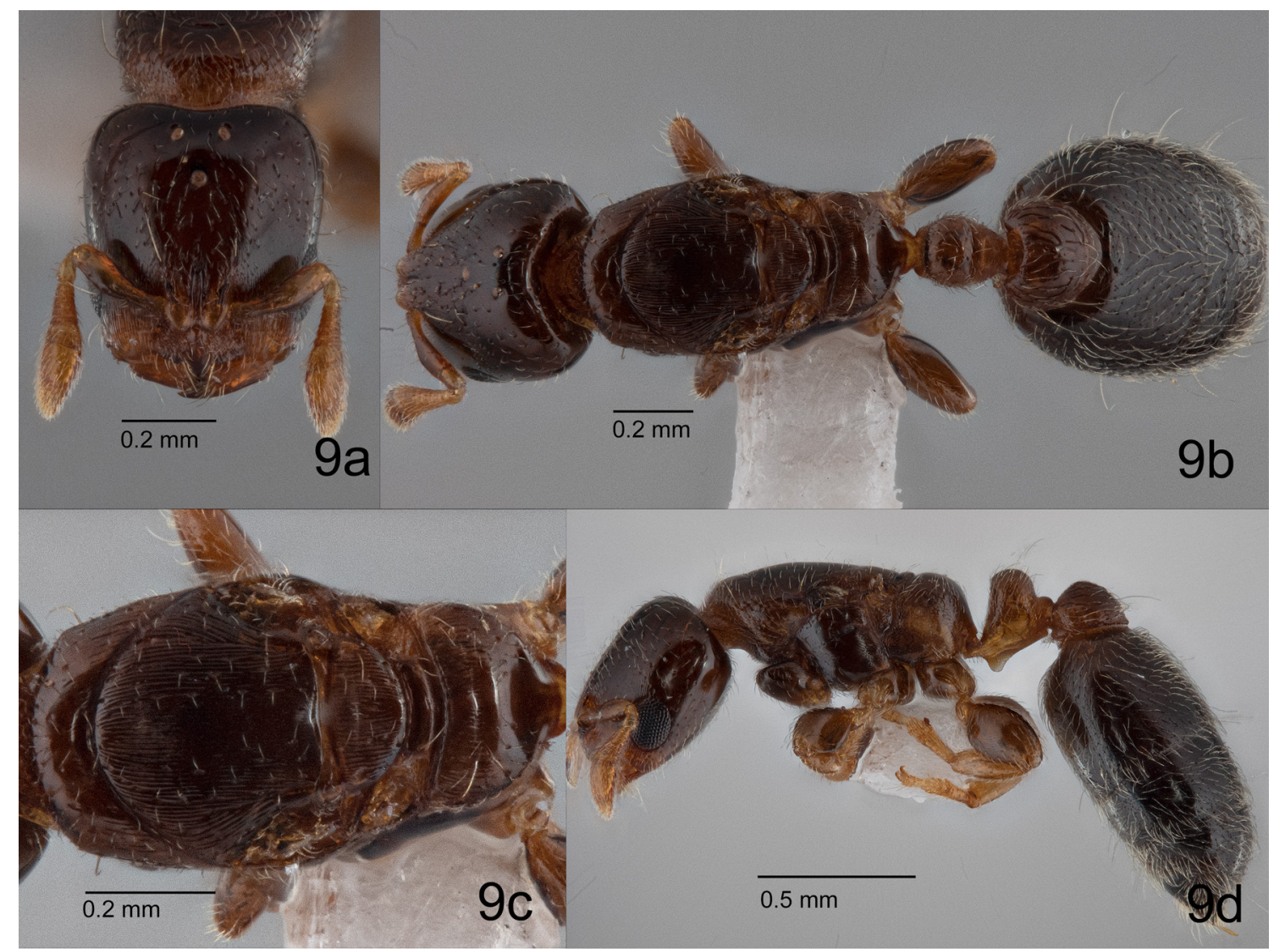

Fig. 9. Rhopalomastix johorensis Wheeler, 1929, non-type, queen, Central Thailand (ZRC_ ENT00013956). a. Head in full-face view. b. Body in dorsal view. c. Close-up of mesosoma in dorsal view. d. Body in lateral view. 
Anterior half of head including frontal lobes largely finely striate and shiny, posterior half of head mostly smooth and shining with scattered punctures, vertexal area with feeble fragmented striations; ventral face of head mostly smooth and shining. Median clypeal triangle coarsely striate-reticulate but less coarse than that of worker, interspaces punctate but shiny. Mandible mostly smooth and shining with very feeble striations. Lateral face of mesosoma weakly striate-reticulate, interspaces smooth and shining (Fig. 9d); dorsum largely finely striate and shiny, median section of pronotum for Thai queens mostly smooth and shiny and less striate than that of Singapore queens (Fig. 9c). Petiole and postpetiole largely superficially reticulate with interspaces smooth and shining; apical faces of petiole and postpetiolar dorsum entirely smooth and shining. Gaster superficially reticulate with interspaces smooth and shining, and scattered punctures.

Dorsal and ventral faces of head with dense short suberect and decumbent hairs, interspersed with sparse long erect hairs; antennal scape with uniform short standing hairs, and two long erect hairs near base and median of scape respectively. Dorsum of mesosoma with sparse short standing hairs (Fig. 9b-c), paired long erect hairs sparsely present along dorsolateral margin, with one pair each flanking the anterior margin of pronotal disc, pronotum, mesoscutum, and propodeum; mesoscutum and mesoscutal disc each one pair of long erect hairs. Lateral face of pronotum with decumbent short hairs, pilose relatively to rest of mesosoma. Petiole and postpetiole with dense short standing hairs, and sparse long erect hairs. Gaster more pilose compared to mesosoma, with dense appressed and decumbent hairs on both dorsal and ventral faces; long erect hairs sparsely present along posterior margins of sternites and tergites.

Body generally uniformly dark-brown; legs lighter brown, becoming progressively paler from femur to tarsus. Antennal funiculus and lateral surfaces of petiole, postpetiole also lighter brown. Generally darker in colour relative to worker.

\section{Male}

Measurements. Non-type males ( $\mathrm{n}=12$ ): EL 0.22-0.26; EW 0.18-0.22; HL 0.40-0.48; HW 0.40-0.46; ML 0.82-1.00; MsW 0.42-0.52; PtH 0.17-0.21; PtL 0.20-0.26; SL 0.08-0.09; TL 2.36-2.80; CI 92-96; PtHI 75-84; REL 55-60; SI 18-23.

Relatively large size. Head in full-face view broad ovate, somewhat narrower posteriorly, posterior margin strongly convex (Fig. 10a). Eye extremely large (20-25 ommatidia in the longest axis), posteriormost point just below transverse midline of head, outline of eye roundly convex and bulging from lateral margin of head in full face view (Fig. 10a). Median ocellus subcircular and less oval than lateral ocelli; small area of head dorsum immediately anterior to median ocellus moderately depressed (Fig. 10a). In lateral view, head broad-ovate and lobular, narrowest at ocelli, dorsum steeply sloping, lateral corners indistinct and rounded, ventral margin rounding into posterior margin as a continuous broadly convex curve (Fig. 10b). In the same view, clypeus projected strongly from anterodorsal margin of head, short dorsal clypeal face transitions to longer anterior face at weakly angular edge, anterior face weakly convex or almost flat (Fig. 10b). In lateral view, mesosoma subcylindrical and elongate, dorsal outline broadly and weakly convex, anterodorsal face of mesoscutum distinctly higher than anterior face of pronotum; propodeal junction roundly angulate, posterior propodeal declivity steep and weakly convex or flat (Fig. 10e). Propodeal dorsum rounding into posterior face at indistinct rounded edge. In posterior view, posterior propodeal face emarginate, rounding into and undifferentiated from lateral propodeal face. In dorsal view, mesosoma obovate, narrower posteriorly, notauli absent, parapsidal line weakly present (Fig. 10c-d). In lateral view, petiole inclined posteriorly, longer than high, petiolar node subtriangular with bluntly rounded apex, apex rounding into posterior petiolar face as a continuous convex slope, anteroventral extension of subpetiolar process reduced to a short and shallow translucent lamella (Fig. 10e). Dorsal outline of postpetiole almost entirely flat, slightly differentiated from gaster by an indistinct cinctus (Fig. 10e). 
Anterior half of head dorsum rugulose-reticulate, interspaces punctate and weakly shining, rest of head mostly substriate-reticulate and shiny with scattered punctures, punctures on posterior face of head denser than rest of head; area enclosed by ocelli rugulose-punctate and weakly shining. Lateral face of mesosoma mostly superficially substriate-reticulate with interspaces smooth and shining (Fig. 10e). In dorsal view, mesoscutum largely finely substriate with interspaces smooth and shining, lateralmost area of mesoscutum in between parapsidal line and parascutellar carina unsculptured, largely smooth and shining; mesoscutal disc largely smooth and shining with feeble superficial striations; propodeum superficially reticulate with interspaces smooth and shining (Fig. 10d). Strength of sculpture on mesoscutum can vary substantially among different Thai populations, both sympatric and allopatric. Petiole, postpetiole and gaster superficially reticulate with interspaces smooth and shining.

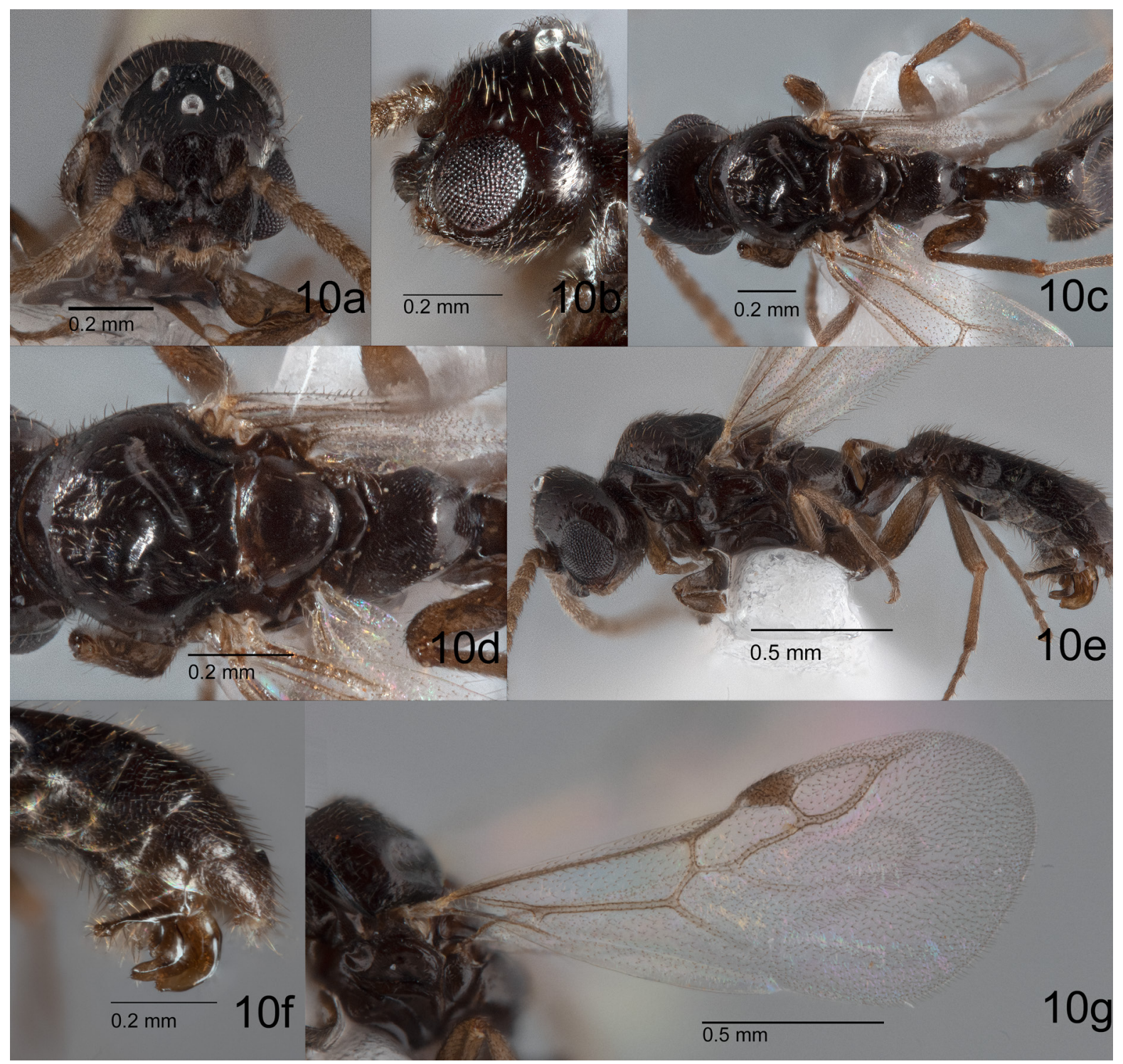

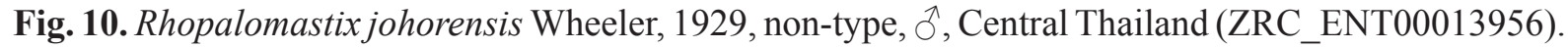
a. Head in full-face view. b. Head in profile. c. Body in dorsal view. d. Close-up of mesosoma in dorsal view. e. Body in lateral view. f. Close-up of genitalia in profile. g. Forewing in lateral view. 
Dorsum of head with fairly dense and uniform short standing hairs, ventral face with dense short standing and decumbent hairs. Dorsum of mesosoma with scattered short standing hairs, most dense on mesoscutum (Fig. 10d); standing hairs on propodeum limited to posterior half. Dorsums of petiole and postpetiole with dense standing hairs. Gaster pilose, with dense standing, decumbent and appressed hairs of generally uniform length.

Body generally dark greyish brown, head more blackish and darker than rest of body; antennae and joints of legs pale light brown.

\section{Distribution and bionomics}

Indonesia (West Java), Singapore, Thailand (Fig. 16b). Colonies in Thailand are often found in, but not limited to, bark of cultivated mango trees usually on roadsides and/or plantations, close to human dwellings. Actual extent of nests per respective host tree is often unclear, sometimes nest cavities have been uncovered in bark very close to tree base, concurrently with occurrence more than one vertical metre from the base. Nests have also been found near or in the crowns of some mango trees. This species has also been collected in bark of wild Azadirachta excelsa trees, in primary evergreen and peat swamp forests of southern Thailand.

\section{Remarks}

There is broad internidal variation in morphology for this species in Thailand, mostly in terms of minor characters that were also observed to vary within different colonies, such as: intensity of striations on posterior half of head, and extent of flatness of anterior clypeal face. Based on COI barcodes, most Thai colonies could be grouped into a large cluster with $<1 \%$ uncorrected $p$ distances among individuals (Fig. 1); colonies collected from East Thailand, however, formed a separate cluster divergent from the rest by $4.5 \%$ (Fig. 1). The East Thai specimens are morphologically indistinguishable from other specimens of $R$. johorensis, despite the relatively high underlying genetic difference between the two clusters. This result may imply that East Thai populations of the species were established via an introduction event different from populations in all other parts of Thailand (Bergsten et al. 2012). Alternatively, there could have been a deep genetic split in Thai $R$. johorensis in the past for reasons unknown, leading to the development of two separate lineages (i.e., East Thai vs all other parts of Thailand) of the same species. More empirical evidence is required to draw a definite conclusion.

This species is similar to both $R$. impithuksai Wang \& Jaitrong sp. nov. and $R$. javana, but can be distinguished from both mainly by the condition of posterolateral corners of propodeum, in relation to inner posterior propodeal face. In $R$. johorensis, posterolateral corners of propodeum are bluntly rounded but ill-defined and not distinctly differentiated from the inner posterior propodeal face; whereas in $R$. impithuksai Wang \& Jaitrong sp. nov. and R. javana, posterolateral corners of propodeum are bluntly angulate, distinctly projecting posteriorly and differentiated from the inner posterior propodeal face, thus the posterior propodeal margin for the latter two species also appears more distinctly (though still weak) concave in dorsal view.

Previously in Wang et al. (2018b), it was surmised that workers of $R$. javana and $R$. johorensis may be differentiated based on strength of sculpture on the posteriormost vertexal strip close to the occiput in posterodorsal view. However, upon examination of additional material from Thailand, it is evident that this character is highly variable within both $R$. javana and $R$. johorensis - the posteriormost vertexal strip can range from being finely striate to almost entirely smooth and shining. Thus, in this revision we do not recommend using this character to distinguish between the two species. 


\section{Rhopalomastix parva Wang \& Jaitrong sp. nov. urn:1sid:zoobank.org:act:AD667B83-8206-429E-8918-C8530BBC46A9 \\ Figs 2e, 11 (worker), 12 (queen), 16a}

\section{Diagnosis}

\section{Worker}

Generally small in size (TL 1.85-2.25), workers monomorphic with little to moderate intranidal size variation. Head in full-face view subrectangular, distinctly longer than wide (Fig. 11a); clypeus in profile strongly projected forward with short downward-sloping and weakly convex dorsal face rounding into steeper anterior face at indistinct edge, outline of anterior face broadly and weakly concave or sometimes almost flat (Fig. 11c). In lateral view, mesosoma elongate subcylindrical; propodeum subtrapezoidal, distinctly longer ventrally than dorsally (Fig. 11d). In dorsal view, anterior margin of pronotal disc strongly convex, roundly angulate at its median point, humeral corners rounded and obtusely angulate; lateral mesosomal margin weakly inflexed just before propodeum; posterolateral corners of propodeum rounded and indistinct, posterior propodeal margin weakly concave and indistinct (Fig. 11b). Posterior face of propodeum very weakly marginate. Petiole suboval in dorsal view, wider than long; postpetiole suboblong, about twice as wide as long (Fig. 11b). Dorsum and lateral face of head finely striate and shiny with scattered punctures, frons and vertex more weakly shining than rest of head dorsum. Median clypeal face mostly finely striate and shiny, with smooth and shining median longitudinal strip. Gaster largely smooth and shining, bases of gastral tergites with feeble superficial reticulation. Head and antennal funiculus mostly with dense short standing and decumbent hairs. Body mostly light brown with head and gaster darker brown; ventral faces of femora and tibiae usually pale yellowish brown.

\section{Queen}

Small size (TL 2.44-2.52). Head in full-face view subrectangular and distinctly longer than wide (CI 8487) (Fig. 12a). Lateral and ventral faces of head roughly differentiated by very weak, indistinct ridge behind eye connecting ventral margin of eye to posterolateral corner of head (Fig. 12d); posterolateral corner of head weakly present as bluntly rounded shallow protrusion next to posterior margin in lateral view. Ocelli small and oval, lateral ocelli closer to each other than to median ocellus (Fig. 12a-b). Propodeal junction indistinct, dorsum of propodeum rounding smoothly into steep, weakly convex posterior propodeal face at indistinct edge (Fig. 12d). In dorsal view, mesosoma subovate and distinctly narrowed posteriorly (Fig. 12b-c). In lateral view, petiolar node sub-trapezoidal, apical dorsum short and weakly convex, rounding into and not distinctly differentiated from posterior face (Fig. 12d). Posterior half of head dorsum mostly with weak striae that are more fragmented closer to posterior margin, interspaces smooth and shining; frons and vertexal area around ocelli consistently finely striate and shiny. Dorsum of mesosoma mostly finely striate and shiny, median section of pronotal dorsum smooth and shining with sparse punctures (Fig. 12c). Posteromedian subtriangular area of propodeal dorsum entirely smooth and shining, continuous with posterior face of propodeum that is also smooth and shining (Fig. 12c). Apices of petiole and postpetiole, gaster entirely smooth and shining. Dorsum of head, lateral and ventral faces with fairly dense short suberect hairs, interspersed with very sparse long erect hairs. Head, most of mesosoma, and gaster dark blackish brown; pronotum lighter greyish brown.

\section{Etymology}

The species name means 'small' in Latin, in reference to the relatively small size of this species.

\section{Material examined}

\section{Holotype}

THAILAND • worker; Central Thailand, Nakhon Nayok Province, Muaeng District, Ban Hin Subdistrict; 14²6'14" N, 101²2'33" E; 927 m a.s.1.; 18 Nov. 2019; W. Wang \& W. Jaitrong leg.; hill evergreen 
forest, nest in bark of large dipterocarp tree, Toona ciliata M. Roem.; colony code: WJW-THL19-006; THNHM-I-21759.

\section{Paratypes}

THAILAND - 20 workers, 2 queens; same collection data as for holotype; THNHM-I-21760 to THNHM-I-21779 (20 w), THNHM-I-21780, THNHM-I-21781 (2 q) • 22 workers, 3 queens; same collection data as for holotype; GenBank: MW267129, MW267130 (2 workers barcoded); ZRC ENT00013948.1 to ZRC_ENT00013948.25.

\section{Other material}

THAILAND • 16 workers, 2 queens; Central Thailand, Nakhon Nayok Province, Muaeng District, Ban Hin Tang Subdistrict; $14^{\circ} 25^{\prime} 53^{\prime \prime}$ N, $101^{\circ} 22^{\prime} 15^{\prime \prime}$ E; 800 m a.s.1.; 18 Nov. 2019; W. Wang leg.; nest in bark of large dipterocarp tree; colony code: WW-KY19-001; THNHM 10 workers, 2 queens; same collection data as for preceding; ZRC_ENT00013951 • 8 workers; same collection data as for preceding; W. Wang \& W. Jaitrong leg.; nest in bark of large dipterocarp tree, Toona ciliata; colony code: WJWTHL19-004; THNHM • 8 workers; same collection data as for preceding; ZRC_ENT00013950.

\section{Description}

\section{Worker}

Measurements. Holotype worker: EL 0.08; EW 0.05; HL 0.47; HW 0.41; ML 0.58; PronW 0.30 PtH 0.18; PtL 0.20; SL 0.18; TL 1.97; CI 87; PtHI 90; REL 20; SI 44.

Paratype workers $(\mathrm{n}=8)$ : EL 0.07-0.10; EW 0.05-0.06; HL 0.45-0.50; HW 0.38-0.42; ML 0.54-0.62; PronW 0.26-0.30; PtH 0.16-0.20; PtL 0.17-0.22; SL 0.18-0.20; TL 1.85-2.25; CI 84-87; PtHI 86-91; REL 19-24; SI 44-48.

Workers monomorphic with little to moderate intranidal variation in size. Head in full-face view subrectangular, distinctly longer than wide, posterior margin with a broad but shallow median concavity, posterolateral corners roundly angulate, lateral margins of head broadly and weakly convex, almost parallel and weakly converging anteriorly, anterior clypeal margin slightly less wide than posterior margin of head, with broadly and weakly convex median section (Fig. 11a). Eye with 11-15 ommatidia. In lateral view, clypeus strongly projected forward with short downward-sloping and weakly convex dorsal face rounding into steeper anterior face at indistinct edge, outline of anterior face broadly and weakly concave or sometimes almost flat (Fig. 11c). In lateral view, mesosoma elongate subcylindrical, dorsal outline weakly and broadly convex, propodeal junction roundly obtuse, propodeal declivity shallowly concave, propodeum subtrapezoidal, distinctly longer ventrally than dorsally (Fig. 11d); dorsum of propodeum differentiated from posterior declivitous face by indistinct rounded edge. In dorsal view, mesosoma subcylindrical, anterior margin of pronotal disc strongly convex, roundly angulate at its median point, humeral corners rounded and obtusely angulate; lateral margins broadly and weakly convex in anterior $2 / 3$, almost parallel to each other, weakly inflexed just before propodeum; lateral margins of propodeum broadly and more strongly convex than anterior $2 / 3$ of lateral mesosomal margin, posterolateral corners rounded and indistinct, posterior propodeal margin weakly concave and indistinct (Fig. 11b). Posterior face of propodeum very weakly marginate, differentiated from lateral propodeal face by indistinct rounded edge. In lateral view, petiole inclined posteriorly, slightly longer than high, distinctly narrower at apex than at base, anterior face weakly concave and longer than posterior face; apex roundly convex, apical dorsum rounding smoothly into steep posterior face at indistinct rounded edge; petiole higher and longer than postpetiole, dorsal margin of postpetiole evenly convex (Fig. 11d). In dorsal view, petiole suboval, wider than long; postpetiole suboblong, about twice as wide as long (Fig. 11b). 
Dorsum and lateral face of head finely and densely striate and shiny with scattered punctures, frons and vertex more weakly shining than rest of head dorsum. Ventral face of posterior half of head mostly superficially substriate-reticulate with interspaces smooth and shining. Mandible mostly smooth and shining, area immediately adjacent to masticatory margin rugulose-punctate (Fig. 2e). Median clypeal face mostly finely striate and shiny, with smooth and shining median longitudinal strip. Lateral face of mesosoma largely superficially substriate-reticulate with interspaces smooth and shining (Fig. 11d); dorsum of mesosoma finely striate and less shiny than lateral mesosomal face (Fig. 11b). Lateral face of petiole superficially reticulate with interspaces smooth and shining (Fig. 11d), petiolar apex finely but weakly striate and shiny (Fig. 11b). Postpetiole largely superficially striate-reticulate with interspaces and dorsomedian spot smooth and shining. Gaster largely smooth and shining, bases of gastral tergites with feeble superficial reticulation.

Dorsum of head with fairly dense though well-spaced short erect and suberect hairs; gena and ventral face of head with fairly dense standing and short subdecumbent hairs; temple just above antennal scrobe, subocular region shortly posterior to eye, frontal carina, and median clypeal face each with a long erect hair. Antennal funiculus with dense standing and decumbent hairs. Mesosoma dorsum largely with sparse, scattered short erect hairs, posterolateral corners of propodeal dorsum with relatively denser standing hairs; dorsolateral margin lined with short standing hairs; paired long erect hairs sparsely present along dorsolateral margin, with one pair each flanking the anterior margin of pronotal disc, pronotum, mesonotum and propodeum respectively. Anterior rising face of petiole with uniformly short

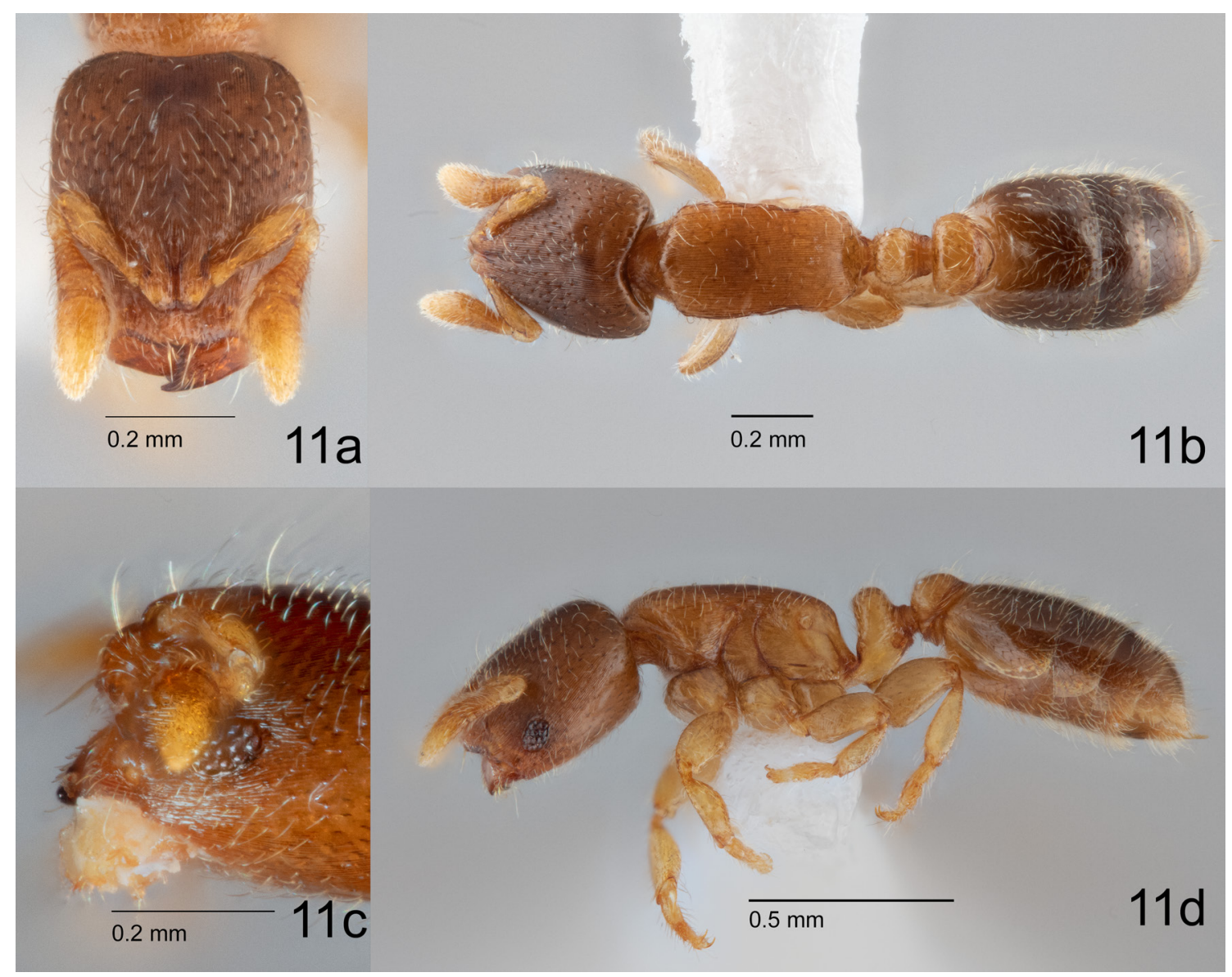

Fig. 11. Rhopalomastix parva Wang \& Jaitrong sp. nov., paratype, worker (ZRC_ENT00013948). a. Head in full-face view. b. Body in dorsal view. c. Close-up of clypeus in profile. d. Body in lateral view. 
standing and decumbent hairs, apex and posterior declivity with dense short standing hairs, apex with one pair of long erect hairs. Postpetiole with dense short erect and suberect hairs, and one pair of long erect hairs. Gaster pilose, with dense suberect and erect hairs that are generally longer and less fine than hairs on mesosoma dorsum.

Body mostly light brown with head and gaster darker brown; tips of antennae, first funicular segment, tarsi, ventral faces of femora and tibiae pale yellowish brown.

\section{Queen}

Measurements. Paratypes, dealate queens $(\mathrm{n}=2)$ : EL 0.14; EW 0.10-0.11; HL 0.46-0.50; HW 0.400.42; ML 0.72-0.80; MsW 0.36-0.40; PtH 0.19-0.21; PtL 0.23-0.24; SL 0.18-0.20; TL 2.44-2.52; CI 84-87; PtHI 83-85; REL 33-35; SI 45-48.

Small size (TL 2.44-2.52). Head in full-face view subrectangular, distinctly longer than wide, slightly narrower anteriorly; lateral margins broadly and weakly convex, almost entirely parallel to each other; posterolateral corners rounded, posterior margin nearly straight with a shallow median concavity (Fig. 12a). Eye very large (13-15 ommatidia in the longest axis); posteriormost point of eye located slightly below

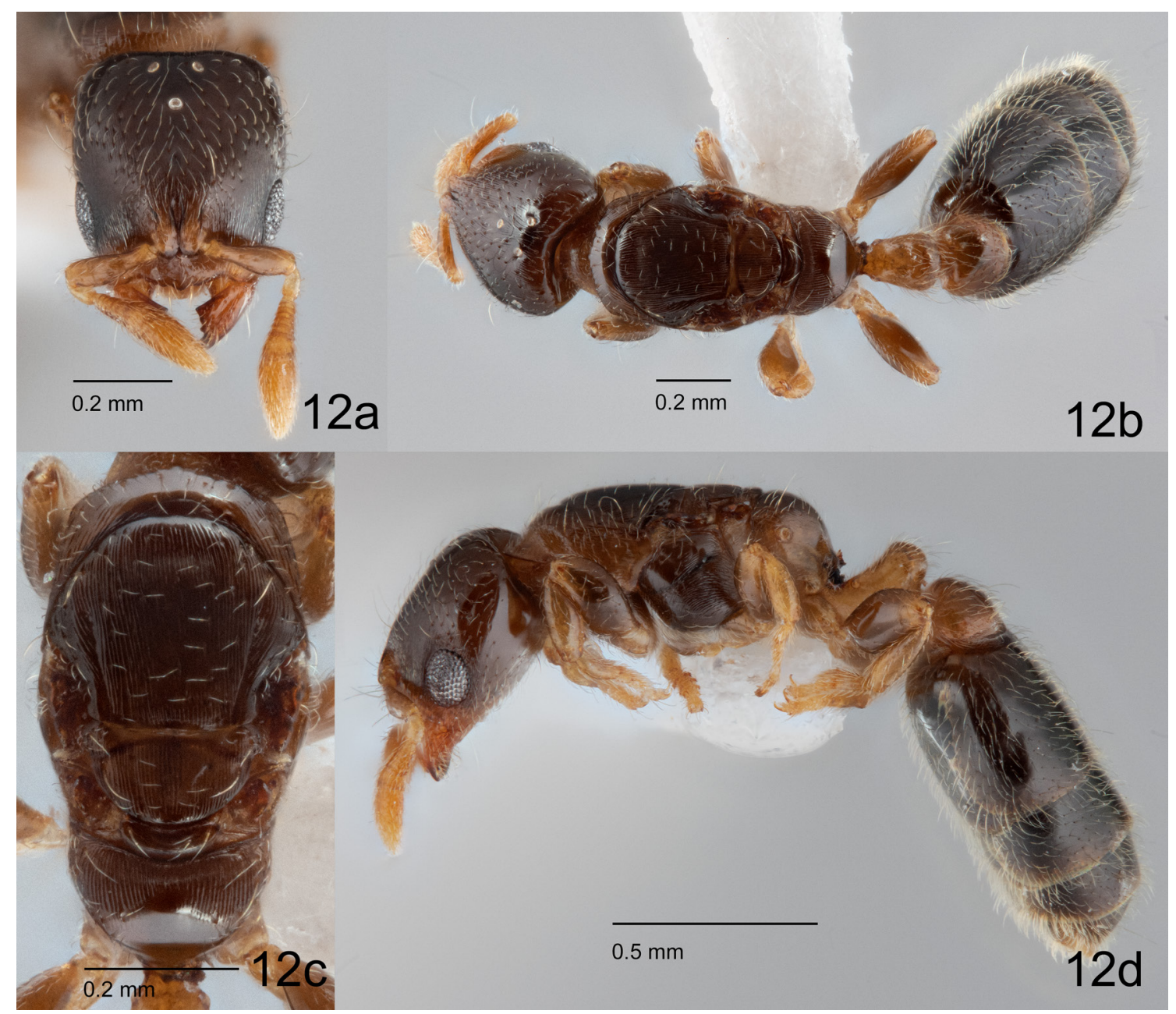

Fig. 12. Rhopalomastix parva Wang \& Jaitrong sp. nov., paratype, queen (ZRC_ENT00013948). a. Head in full-face view. b. Body in dorsal view. c. Close-up of mesosoma in dorsal view. d. Body in lateral view. 
transverse midline of head in full-face view (Fig. 12a). In lateral view, clypeus weakly and broadly convex; head behind eye with very weak, indistinct ridge connecting ventral margin of eye to posterolateral corner of head, roughly differentiating lateral and ventral faces of head (Fig. 12d); posterolateral corner of head weakly present as bluntly rounded shallow protrusion next to posterior margin. Ocelli small and oval, median ocellus slightly larger than lateral ocelli, lateral ocelli slightly closer to each other than to median ocellus (Fig. 12a-b); small area immediately anterior to median ocellus weakly depressed. In lateral view, mesosoma subcylindrical, dorsal outline weakly and broadly convex, propodeal junction indistinct (Fig. 12d); dorsum of propodeum rounding smoothly into steep, weakly convex posterior propodeal face at indistinct edge. In dorsal view, mesosoma subobovate, distinctly narrowed posteriorly; anterior margin of pronotal disc broadly convex, humeral corners obtusely angulate, mesoscutum wider than long; propodeum much wider than long, posterior margin weakly present and indistinct, lateral propodeal margin broadly convex, rounding into posterior margin at indistinct rounded edge (Fig. 12b). In lateral view, petiole inclined posteriorly, longer than high, anterior petiolar face weakly concave and steep; petiolar node subtrapezoidal, apical dorsum short and weakly convex, rounding into and not distinctly differentiated from weakly convex posterior face; posterior face shorter and steeper than anterior face (Fig. 12d). In lateral view, postpetiole not as long as petiole, dorsal margin broadly and weakly convex (Fig. 12d). In dorsal view, petiole suboval, less than twice as wide as long; postpetiole globular, less than twice as wide as long.

Anterior half of head dorsum finely striate and shiny, posterior half mostly with weaker striae that are more fragmented closer to posterior margin, interspaces smooth and shining; frons and vertexal area around ocelli consistently finely striate and shiny. Clypeus mostly finely striate and shiny, with a distinct median longitudinal strip that is smooth and shining. Lateral and ventral faces of head mostly smooth and shining. Mandible mostly smooth and shining, area next to masticatory margin densely striatepunctate. Lateral face of mesosoma mostly weakly substriate-reticulate with interspaces smooth and shining; anepisternum smooth and shining with sparse fragmented striae. Dorsum of mesosoma mostly finely striate and shiny, median section of pronotal dorsum smooth and shining with sparse punctures; lateral-most triangular area of mesoscutum immediately above parascutellar carina more superficially striate than rest of mesoscutum, with smooth and shining interspaces; posteromedian subtriangular area of propodeal dorsum entirely smooth and shining, continuous with posterior face of propodeum that is also smooth and shining (Fig. 12c). Lateral faces of petiole and postpetiole superficially reticulate with interspaces smooth and shining (Fig. 12d), apicodorsal faces almost entirely smooth and shining with sparse punctures. Gaster largely smooth and shining.

Dorsum of head, lateral and ventral faces with fairly dense short suberect hairs, interspersed with very sparse long erect hairs, including one pair on median section of clypeus. Antennal scape with uniform short standing hairs and a long erect hair on basal and median points respectively. Dorsum of mesosoma with sparse short standing or decumbent hairs, more dense closer to dorsolateral margin (Fig. 12cd); paired long erect hairs sparsely present along dorsolateral margin, with one pair each flanking the anterior margin of pronotal disc, pronotum, mesoscutum, and propodeum; mesoscutum and mesoscutal disc each one pair of long erect hairs. Petiole entirely with dense short standing hairs, apex with one pair of long erect hairs. Postpetiole with dense short standing hairs and 1-2 pairs of long erect hairs. Gaster pilose, with dense short standing hairs, mostly longer than those on head and mesosoma, interspersed with long erect hairs sparsely distributed along posterior margins of gastral tergites.

Head, most of mesosoma, and gaster dark blackish brown; antennal scape, pronotum, femora, petiole and postpetiole lighter greyish brown. Tips of antennae, tarsi and joints of legs pale yellowish brown.

\section{Distribution and binomics}

Thailand (Fig. 16a). Colonies of this species may be found in bark of Toona ciliata near edges of generally good secondary forest, at moderately high elevation $(800-927 \mathrm{~m})$. 
Rhopalomastix robusta Wang \& Jaitrong sp. nov. urn:lsid:zoobank.org:act:7FE9D47B-988F-40BB-9AEE-C208F52D6159

Figs 2b, 13 (average worker), 14 (large worker), 15 (small worker), 16a

\section{Diagnosis}

\section{Worker}

Relatively large size (small worker TL $>2 \mathrm{~mm}$; large worker TL $>3 \mathrm{~mm}$ ) with robust habitus; workers monomorphic with broad intranidal size variation. Head subsquarish, as long as wide or slightly wider than long; in small workers slightly longer than wide; distinctly narrowed anteriorly; narrow median furrow between frontal lobes deep and long, extending till or past transverse midline of head, narrower or constricted shortly before midlength; posterolateral corners roundly convex and laterally expanded forming somewhat rounded vertexal lobes (Figs 13a, 14a, 15a). Anterior clypeal face in profile weakly and evenly convex, sometimes almost flat (Fig. 13c). In dorsal view, anterior margin of pronotal disc broadly convex with bluntly angulate median point, humeral corners obtusely angulate, lateral margins with distinct inflexions differentiating pronotum, mesonotum and propodeum; posterolateral corners of propodeum indistinct and rounded; posterior propodeal margin indistinctly concave (Figs 13b, 14b, $15 \mathrm{~b}$ ). Posterior face of propodeum very weakly marginate, rounding smoothly into lateral propodeal face without distinct marginate edge. Median clypeal face coarsely carinulate, interspaces punctate and weakly shiny. Mandible with upper half of outer face finely striate, lower half superficially substriate (Fig. 2b). Petiolar node and postpetiole each with two pairs of very long erect hairs. In larger workers, head, dorsum of mesosoma, and procoxa blackish or dark reddish brown, with rest of body generally uniformly lighter brown, tips of antennae and legs pale yellowish brown.

\section{Etymology}

The species name is a both a direct reference to the robust habitus of the workers, and also a subtle allusion to the species' intense dark brown colour resembling brewed robusta coffee.

\section{Material examined}

\section{Holotype}

THAILAND • worker; Central Thailand, Nakhon Nayok Province, Muaeng District, Ban Hin Subdistrict; $14^{\circ} 26^{\prime} 14^{\prime \prime} \mathrm{N}, 101^{\circ} 22^{\prime} 33^{\prime \prime}$ E; 927 m a.s.1.; 8 Jan. 2018; W. Jaitrong leg.; hill evergreen forest, nest in bark of Toona ciliata M. Roem.; colony code: WJT080118-1; THNHM-I-21782.

\section{Paratypes}

THAILAND • 19 workers; same collection data as for holotype; THNHM-I-21783 to THNHM-I-21801 - 10 workers; same collection data as for holotype; 18 Nov. 2019; W. Wang \& W. Jaitrong leg.; hill evergreen forest, nest in bark of Toona ciliata; colony code: WJW-THL19-007; THNHM - 77 workers; same collection data as for preceding; GenBank: MW267049 to MW267051 (3 barcoded); ZRC_ ENT00013949.1 to ZRC_ENT00013949.77.

\section{Description}

\section{Worker}

Measurements. Holotype worker: EL 0.12; EW 0.08; HL 0.64; HW 0.66; ML 0.86; PronW 0.46; PtH 0.25; PtL 0.27; SL 0.28; TL 2.91; CI 103; PtHI 93; REL 18; SI 42.

Paratype workers $(\mathrm{n}=12)$ : EL 0.09-0.14; EW 0.06-0.08; HL 0.55-0.68; HW 0.52-0.70; ML 0.68-0.96; PronW 0.36-0.48; PtH 0.19-0.28; PtL 0.20-0.31; SL 0.24-0.31; TL 2.24-3.16; CI 95-103; PtHI 91-96; REL 17-20; SI 42-46. 
Relatively large sized for Rhopalomastix (TL 2.24-3.16) with habitus generally robust; workers monomorphic with broad size variation. Head in full-face view subsquarish, as long as wide or slightly wider than long (CI 100-103), in small workers sometimes slightly longer than wide (Fig. 15a), distinctly narrowed anteriorly; narrow median furrow between frontal lobes deep and long, extending till or past transverse midline of head, narrower or constricted shortly before midlength; posterior margin with a broad but shallow median concavity, posterolateral corners roundly convex and laterally expanded to form somewhat rounded vertexal lobes, lateral margins of head broadly convex and weakly converging anteriorly, anterior clypeal margin with broadly and weakly convex median section (Figs 13a, 14a, 15a). Eye with 16-20 ommatidia in small workers, 22-32 ommatidia in larger workers. In lateral view, anterior face of clypeus weakly and evenly convex, sometimes almost flat (Fig. 13c). Mesosoma in lateral view box-shaped and subcylindrical, dorsal outline weakly and broadly convex, almost flat, propodeal junction roundly obtuse, propodeal declivity steep and shallowly concave (Figs 13d, 14c, $15 \mathrm{c})$; propodeal dorsum rounding smoothly into posterior declivitous face at indistinct edge. In dorsal view, mesosoma subcylindrical, anterior margin of pronotal disc broadly convex with bluntly angulate median point, humeral corners obtusely angulate, lateral margins with distinct inflexions differentiating pronotum, mesonotum and propodeum (Figs 13b, 14b, 15b); in large workers, mesosoma weakly narrowed posteriorly (Fig. 14b); lateral margins of propodeum broadly convex with indistinct rounded posterolateral corners, posterior propodeal margin weakly defined (Figs 13b, 14b, 15b). Posterior face of propodeum very weakly marginate and almost flat, sometimes with upper half shallowly depressed,

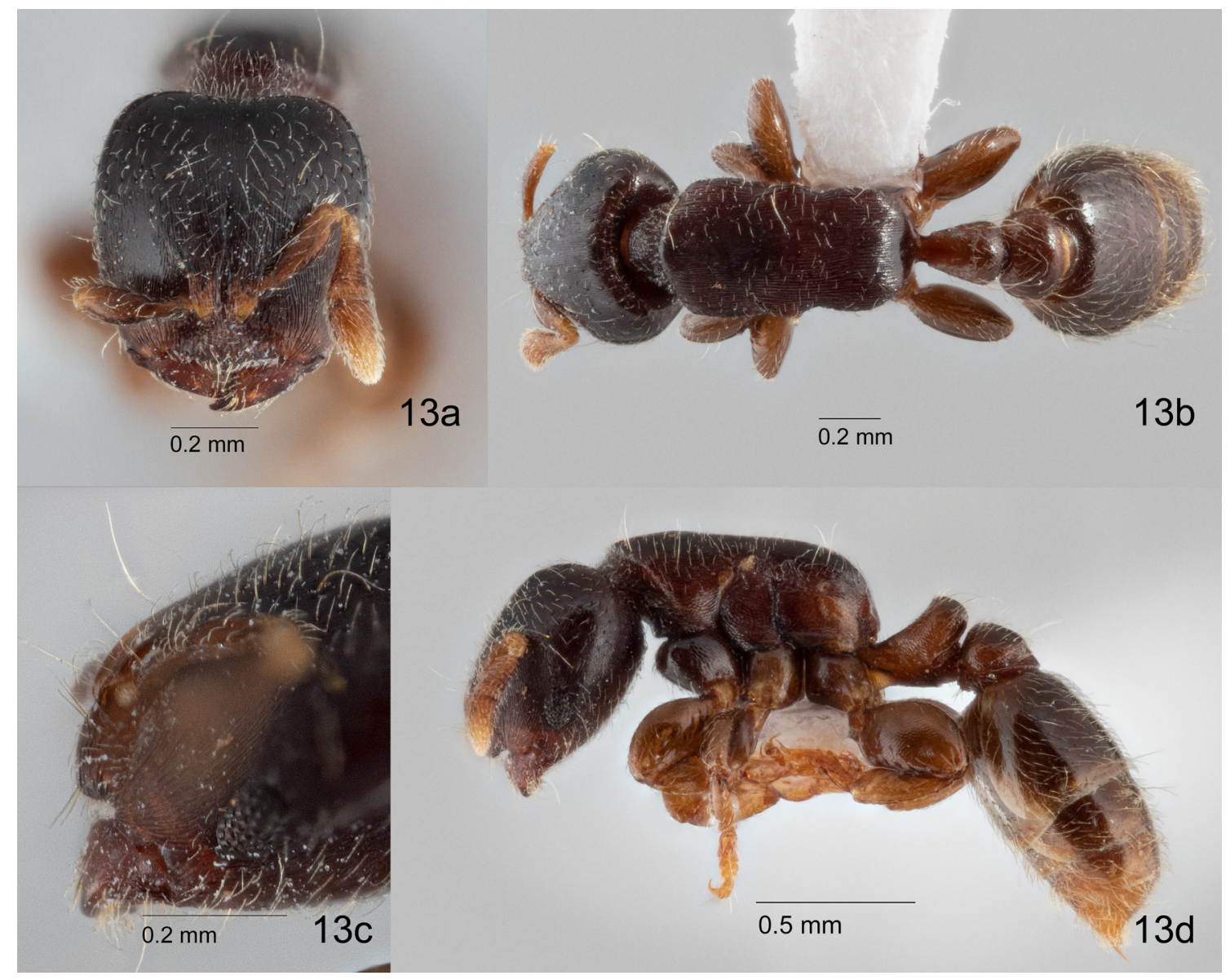

Fig. 13. Rhopalomastix robusta Wang \& Jaitrong sp. nov., paratype, average-sized worker (ZRC_ ENT00013949). a. Head in full-face view. b. Body in dorsal view. c. Close-up of clypeus in profile. d. Body in lateral view. 
rounding smoothly into lateral propodeal face without distinct marginate edge. In lateral view, petiole inclined posteriorly and bell-shaped, slightly longer than high, anterior face steep and weakly concave, apex rounded and slightly higher than postpetiole, apical dorsum rounding into weakly convex posterior face at indistinct rounded edge, posterior face steeper and shorter than anterior face (Figs 13d, 14c, 15c). Petiole higher and longer than postpetiole, dorsal margin of postpetiole weakly convex, almost flat in large workers (Fig. 14c). In dorsal view, petiole suboval, wider than long; postpetiole globular, less than twice as wide as petiole (Figs 13b, 14b, 15b).

Dorsum of head finely striate and dully shining with scattered punctures, lateral face substriate-reticulate with scattered punctures and interspaces smooth and shining; ventral face largely finely reticulate with interspaces smooth and shining; median clypeal face coarsely carinulate, interspaces punctate and weakly shiny. Mandible in full-face view with upper half of outer face finely striate, lower half superficially substriate (Fig. 2b), in larger workers striae on mandible more coarse, area immediately adjacent to masticatory margin punctate. Lateral face of mesosoma largely substriate-reticulate with interspaces smooth and shining (Figs 13d, 14c, 15c); dorsum of mesosoma finely striate and shiny (Figs 13b, 14b, $15 \mathrm{~b}$ ); posterior face of propodeum superficially and transversely substriate-reticulate with interspaces smooth and shining. Coxae and femora distinctly but superficially reticulate with interspaces smooth and shining. Lateral faces of petiole and postpetiole superficially substriate-reticulate with interspaces smooth and shining, apicodorsal faces largely superficially reticulate with interspaces smooth and

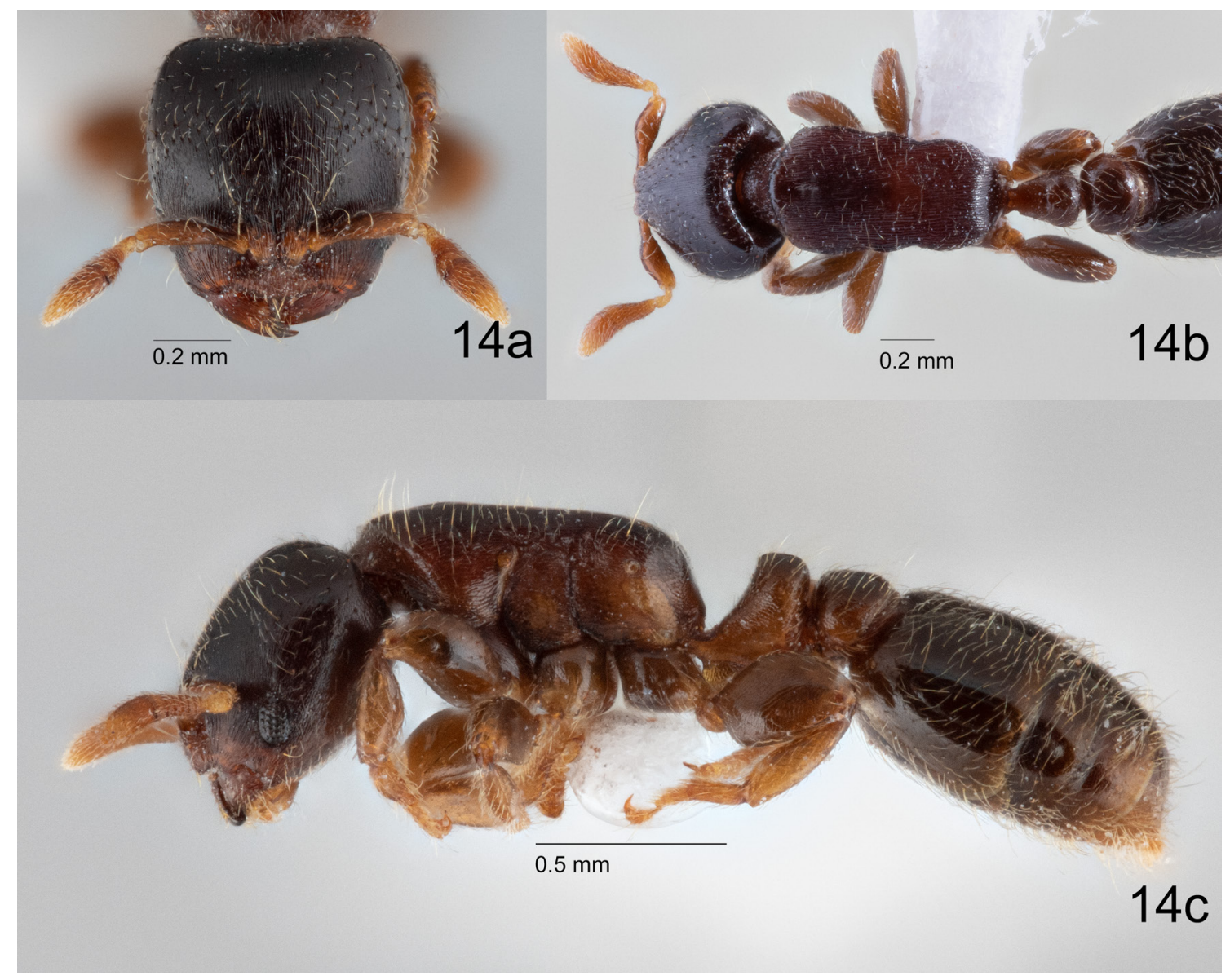

Fig. 14. Rhopalomastix robusta Wang \& Jaitrong sp. nov., paratype, large worker (ZRC_ENT00013949). a. Head in full-face view. b. Body in dorsal view. c. Body in lateral view. 
shining, apicalmost point of petiole entirely smooth and shining. Gaster largely superficially reticulate with interspaces smooth and shining; sculpture slightly stronger around base of first gastral tergite where it articulates with postpetiole.

Dorsum and ventral face of head with fairly dense though well-spaced short standing and suberect hairs interspersed with sparse long erect hairs (Figs 13a, 14a, 15a); lateral face with short suberect and subdecumbent hairs. Mesosoma dorsum with scattered short standing hairs, more dense close to dorsolateral margin (Figs 13b, 14b, 15b); paired long erect hairs sparsely present along dorsolateral margin, with at least one pair each flanking the anterior margin of pronotal disc, pronotum, mesonotum and propodeum; large workers sometimes with 2-3 pairs of long erect hairs on anterior margin of pronotal disc, and/or 2-3 pairs of long erect hairs flanking pronotum and propodeum. Anterior rising

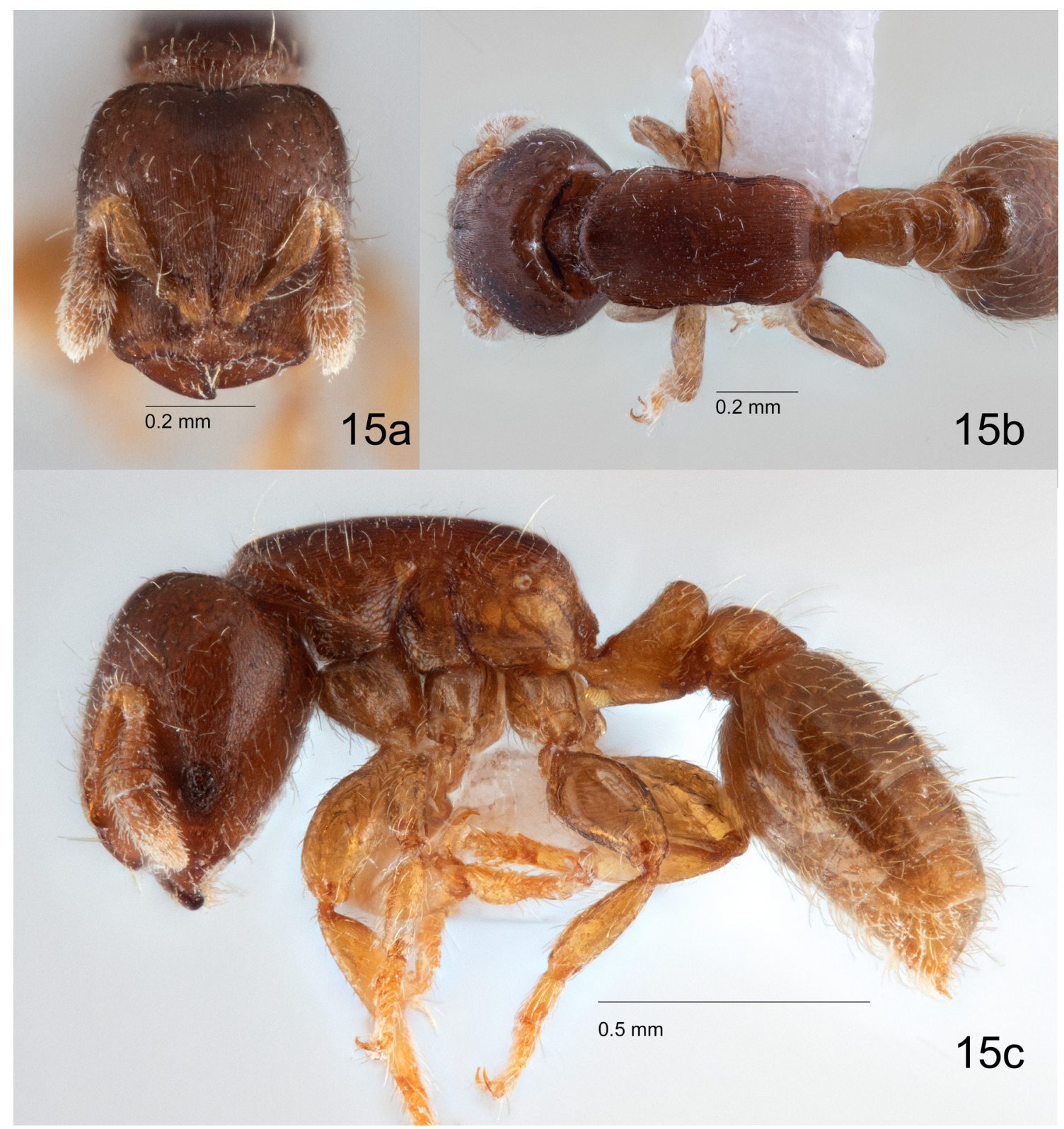

Fig. 15. Rhopalomastix robusta Wang \& Jaitrong sp. nov., paratype, small worker (ZRC_ENT00013949). a. Head in full-face view. b. Body in dorsal view. c. Body in lateral view. 
face of petiole with relatively sparse (compared to head) short decumbent and subdecumbent hairs, slightly more dense closer to apex; lateral petiolar face with 1-2 very long erect hairs and standing hairs slightly longer than those on anterior face, mostly densely along posterolateral margin; apex of petiolar node with sparse standing hairs longer than those on anterior face, and two pairs of very long erect hairs; posterior petiolar face almost hairless or with sparse short standing hairs. Postpetiole with fairly dense erect and suberect hairs longer than those on anterior petiolar face, and two pairs of much longer erect hairs. Gaster pilose with dense erect and suberect hairs, interspersed with sparse long erect hairs.

Head and dorsum of mesosoma generally dark brown with rest of body mostly uniformly lighter brown. In larger workers, head, dorsum of mesosoma, and procoxa blackish or dark reddish brown; petiolar apex and dorsum of postpetiole generally darker brown than their respective lateral faces. Tips of antennae and legs pale yellowish-brown.

\section{Distribution and binomics}

Thailand (Fig. 16a). This species is thus far only known from its type series, collected from relatively good secondary forest at moderately high elevation $(927 \mathrm{~m})$. The colony was found in bark of a large dipterocarp tree, Toon a ciliata, located near the edge of the forest. Members of the colony seemed rather gregarious when found, with numerous individuals moving quickly en masse as a collective group to seek cover when exposed upon removal of a bark layer.

\section{Remarks}

Smaller workers of $R$. robusta Wang \& Jaitrong sp. nov. are similar to large workers of both $R$. impithuksai Wang \& Jaitrong sp. nov. and $R$. johorensis. The first two species can be differentiated by the following: 1) striations on dorsum of head uniformly dense from frontal lobes to posterior margin in R. robusta Wang \& Jaitrong sp. nov., but in R. impithuksai Wang \& Jaitrong sp. nov., striations on head dorsum are weaker and more fragmented in posterior half; 2) outline of clypeus in lateral view-entirely flat or weakly convex with no angulate edge in $R$. robusta Wang \& Jaitrong sp. nov., but with short weakly convex

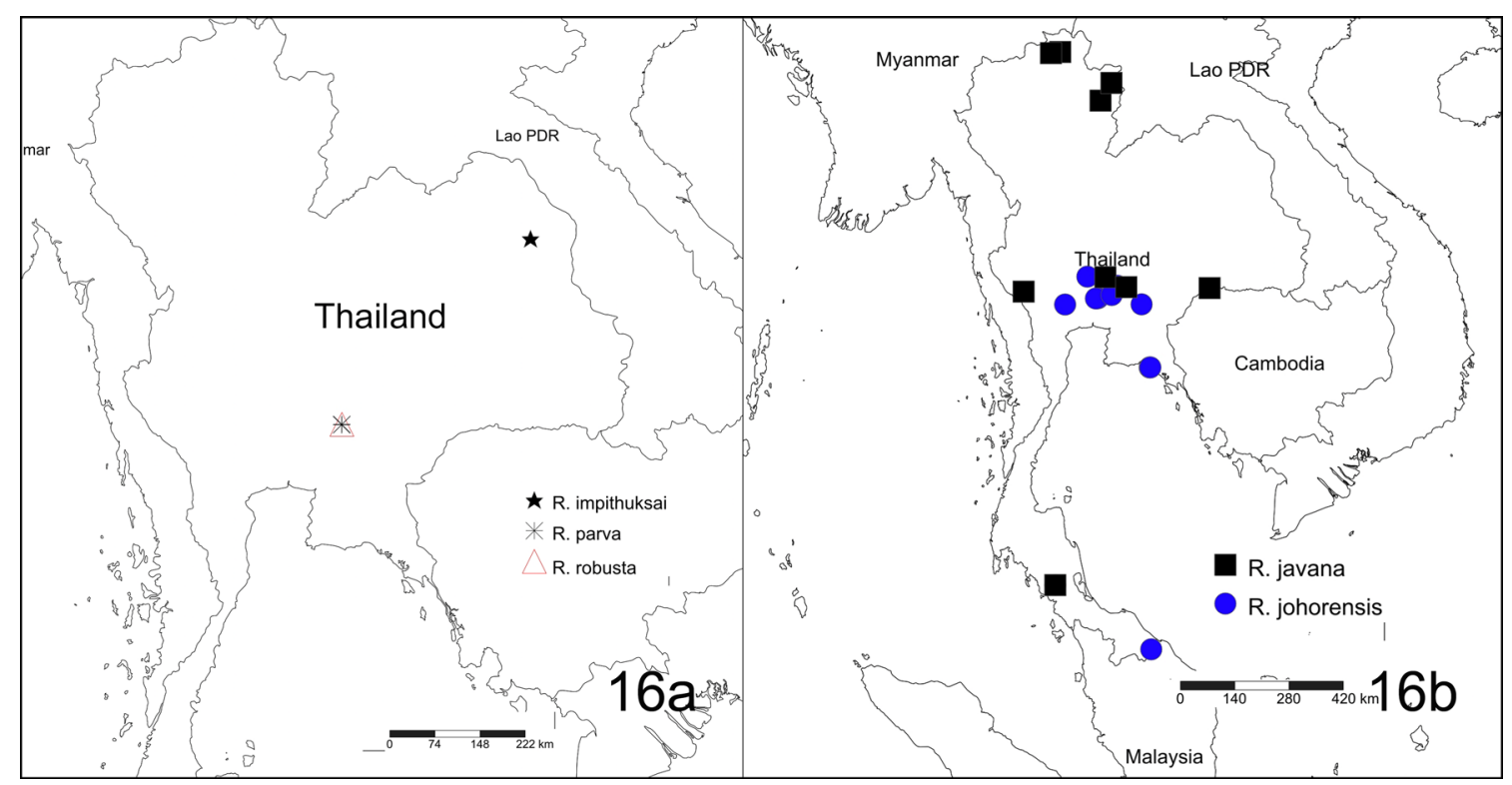

Fig. 16. Distribution maps of species of Rhopalomastix Forel, 1900 in Thailand. a. New species: $R$. impithuksai Wang \& Jaitrong sp. nov., $R$. parva Wang \& Jaitrong sp. nov., R. robusta Wang \& Jaitrong sp. nov. b. R. javana Wheeler, 1929 and $R$. johorensis Wheeler, 1929. 
dorsal face separated from steep and almost flat anterior face by a weak angulate edge in R. impithuksai Wang \& Jaitrong sp. nov.; 3) head in full-face view as long as wide or sometimes wider than long for $R$. robusta Wang \& Jaitrong sp. nov., whereas for $R$. impithuksai Wang \& Jaitrong sp. nov., head in fullface view always longer than wide; 4) median furrow on head dorsum starting from between frontal lobes quite long and relatively deep in $R$. robusta Wang \& Jaitrong sp. nov., either extending till or past transverse midline of head, in $R$. impithuksai Wang \& Jaitrong sp. nov. the median furrow is shorter, usually narrower and shallower in its posterior half, often fading out before transverse midline of head. Points (3) and (4) can also be used to distinguish between $R$. robusta Wang \& Jaitrong sp. nov. (small workers) and $R$. johorensis (large workers).

\section{Discussion}

In this present paper, we have established five species of Rhopalomastix in Thailand, with three species new to science. All Thai species here can be considered to belong to the broad $R$. rothneyi species group, according to the general characters defined in Wang et al. (2018b), i.e., head, femora and most other body surfaces sculptured (usually striate or superficially reticulate to varying degrees), never entirely smooth and shining. More recent new material collected from Thailand has revealed the existence of multiple species, some possibly new, belonging to the $R$. murphyi group (W. Jaitrong pers. obs.), but these have not been thoroughly verified at this point in time. Nevertheless, the $R$. murphyi group species in Thailand will be accounted for in a more comprehensive revision of the genus in Southeast Asia, slated for future conception by the same authors of this paper.

With the additional material from Thailand and three new species, it has become increasingly evident that differentiating between Rhopalomastix species based on explicit external morphology alone is tricky. Differences between species are often subtle - all species have the same generalized morphology without additional conspicuous structural features such as spines or tubercles. The fact that intra-species morphological variation typically runs the gamut of ill-defined intermediate forms further exacerbates the problem of species resolution. Molecular data such as DNA barcodes can help mitigate this issue, but only to a certain extent; validating putative species distinguished by DNA ultimately also relies on morphology. It was under these circumstances that we could not confidently establish the species identities of specimens tentatively treated as $R$. javana in this study.

We do, however, observe that differences between Rhopalomastix species manifest heavily in terms of broad body forms and shapes, which cannot be adequately or accurately characterized by plain description. Instead, methods involving geometric morphometry may need to be employed to empirically quantify such characters, discriminating between species after taking into account both intra- and inter-specific variation. While beyond the scope of the current paper, geometric morphometrics will certainly be key towards providing clearer species delimitation in a future larger revision of the genus Rhopalomastix in Southeast Asia.

\section{Acknowledgements}

We thank Prof. Rudolf Meier for facilitating DNA barcoding of specimens in this study by allowing inclusion of our samples onto high throughput NGS sequencing runs. We also thank Jayanthi, Wanting and Arina of Meier Lab for assistance with sequencing logistics and initial processing of NGS sequence data. Finally, we thank all friends and colleagues who have helped with specimen collection outfield, and reviewers for constructive comments and criticism that helped us improve on the manuscript.

\section{References}

Altschul S.F., Gish W., Miller W., Myers E.W. \& Lipman D.J. 1990. Basic local alignment search tool. Journal of Molecular Biology 215 (3): 403-410. https://doi.org/10.1016/S0022-2836(05)80360-2 
Benson D.A., Cavanaugh M., Clark K., Karsch-Mizrachi I., Lipman D.J., Ostell J. \& Sayers E.W. 2013. GenBank. Nucleic Acids Research 41 (D1): D36-D42. https://doi.org/10.1093/nar/gks1195

Bergsten J., Bilton D.T., Fujisawa T., Elliott M., Monaghan M.T., Balke M., Hendrich L., Geijer J., Herrmann J., Foster G.N. \& Ribera I. 2012. The effect of geographical scale of sampling on DNA barcoding. Systematic Biology 61 (5): 851-869. https://doi.org/10.1093/sysbio/sys037

Billen J. \& Peeters C. 2020. Glandular innovations for a tunnelling life: silk and associated leg glands in Melissotarsus and Rhopalomastix queen and worker ants. Arthropod Structure \& Development 59: 100979. https://doi.org/10.1016/j.asd.2020.100979

Bolton B. 1995. A New General Catalogue of the Ants of the World. Harvard University Press, Cambridge, MA.

Bolton B. 2003. Synopsis and classification of Formicidae. Memoirs of the American Entomological Institute 71: 1-370.

Chapman J.W. \& Capco S.R. 1951. Check list of the ants (Hymenoptera: Formicidae) of Asia. Monographs of the Institute of Science and Technology, Manila 1: 1-327

Donisthorpe H. 1936. Rhopalomastix janeti (Hym. Formicidae) a species of ant new to science. Entomologist's Record and Journal of Variation 48: 55-56. https://doi.org/10.5281/zenodo.26445

Emery C. 1914. Intorno alla classificazione dei Myrmicinae. Rendiconti delle Sessioni della Reale Accademia delle Scienze dell'Istituto di Bologna. Classe di Scienze Fisiche (n.s.) 18: 29-42.

Emery C. 1922. Hymenoptera. Fam. Formicidae. Subfam. Myrmicinae. [part]. In: Wytsmann P. (Dir.) Genera Insectorum 174B: 95-206. L. Desmet-Verteneuil, Brussels.

Forel A. 1900. Un nouveau genre et une nouvelle espèce de Myrmicide. Annales de la Société entomologique de Belgique 44: 24-26.

Forel A. 1917. Cadre synoptique actuel de la faune universelle des fourmis. Bulletin de la Société Vaudoise des Sciences naturelles 51: 229-253. https://doi.org/10.5281/zenodo.25600

Katoh K. \& Standley D.M. 2013. MAFFT multiple sequence alignment software version 7: improvements in performance and usability. Molecular Biology and Evolution 30 (4): 772-780. https://doi.org/10.1093/molbev/mst010

Kranzfelder P., Ekrem T. \& Stur E. 2016. Trace DNA from insect skins: a comparison of five extraction protocols and direct PCR on chironomid pupal exuviae. Molecular Ecology Resources 16 (1): 353-363. https://doi.org/10.1111/1755-0998.12446

Meier R., Shiyang K., Vaidya G. \& Ng P.K. 2006. DNA barcoding and taxonomy in Diptera: a tale of high intraspecific variability and low identification success. Systematic Biology 55 (5): 715-728. https://doi.org/10.1080/10635150600969864

Meier R., Zhang G. \& Ali F. 2008. The use of mean instead of smallest interspecific distances exaggerates the size of the "barcoding gap" and leads to misidentification. Systematic Biology 57 (5): 809-813. https://doi.org/10.1080/10635150802406343

Peeters C., Foldi I., Matile-Ferrero D. \& Fisher B.L. 2017. A mutualism without honeydew: what benefits for Melissotarsus emeryi ants and armored scale insects (Diaspididae)? PeerJ 5: e3599. https://doi.org/10.7717/peerj.3599

Tamura K., Stecher G., Peterson D., Filipski A. \& Kumar S. 2013. MEGA6: molecular evolutionary genetics analysis version 6.0. Molecular Biology and Evolution 30 (12): 2725-2729. https://doi.org/10.1093/molbev/mst197 
Wang W.Y., Srivathsan A., Foo M., Yamane S.K. \& Meier R. 2018a. Sorting specimen-rich invertebrate samples with cost-effective NGS barcodes: validating a reverse workflow for specimen processing. Molecular Ecology Resources 18 (3): 490-501. https://doi.org/10.1111/1755-0998.12751

Wang W.Y., Yong G.W. \& Jaitrong W. 2018b. The ant genus Rhopalomastix (Hymenoptera: Formicidae: Myrmicinae) in Southeast Asia, with descriptions of four new species from Singapore based on morphology and DNA barcoding. Zootaxa 4532 (3): 301-340. https://doi.org/10.11646/zootaxa.4532.3.1

Ward P.S., Brady S.G., Fisher B.L. \& Schultz T.R. 2015. The evolution of myrmicine ants: phylogeny and biogeography of a hyperdiverse ant clade (Hymenoptera: Formicidae). Systematic Entomology 40 (1): 61-81. https://doi.org/10.1111/syen. 12090

Wheeler W.M. 1910. Ants: their Structure, Development and Behavior. Columbia University Press, New York.

Wheeler W.M. 1922. Ants of the American Museum Congo Expedition. A contribution to the myrmecology of Africa. VII. Keys to the genera and subgenera of ants. Bulletin of the American Museum of Natural History 45: 631-710.

Wheeler W.M. 1929. The ant genus Rhopalomastix. Psyche 36 (2): 95-101.

Xu Z. 1999. Systematic studies on the ant genera of Carebara, Rhopalomastix and Kartidris in China (Hymenoptera: Formicidae: Myrmicinae). Acta Biologica Plateau Sinica 14: 129-136.

Yong G., Matile-Ferrero D. \& Peeters C. 2019. Rhopalomastix is only the second ant genus known to live with armoured scale insects (Diaspididae). Insectes Sociaux 66 (2): 273-282. https://doi.org/10.1007/s00040-019-00686-z

Manuscript received: 17 August 2020

Manuscript accepted: 22 December 2020

Published on: 17 March 2021

Topic editor: Gavin Broad

Topic editor: Nesrine Akkari

Desk editor: Pepe Fernández

Printed versions of all papers are also deposited in the libraries of the institutes that are members of the EJT consortium: Muséum national d'histoire naturelle, Paris, France; Meise Botanic Garden, Belgium; Royal Museum for Central Africa, Tervuren, Belgium; Royal Belgian Institute of Natural Sciences, Brussels, Belgium; Natural History Museum of Denmark, Copenhagen, Denmark; Naturalis Biodiversity Center, Leiden, the Netherlands; Museo Nacional de Ciencias Naturales-CSIC, Madrid, Spain; Real Jardín Botánico de Madrid CSIC, Spain; Zoological Research Museum Alexander Koenig, Bonn, Germany; National Museum, Prague, Czech Republic. 